\title{
Turbulent swirling flow in a dynamic model of a uniflow- scavenged two-stroke engine
}

\section{Journal Article}

Author(s):

Ingvorsen, Kristian M.; Meyer, Knud Erik; Walther, Jens Honoré (i); Mayer, Stefan

Publication date:

2014-05-22

Permanent link:

https://doi.org/10.3929/ethz-b-000087231

Rights / license:

In Copyright - Non-Commercial Use Permitted

Originally published in:

Experiments in Fluids 55(6), https://doi.org/10.1007/s00348-014-1748-y 


\title{
Turbulent swirling flow in a dynamic model of a uniflow-scavenged two-stroke engine
}

\author{
K. M. Ingvorsen • K. E. Meyer · J. H. Walther • \\ S. Mayer
}

Received: 19 August 2013/Revised: 28 April 2014/ Accepted: 7 May 2014/Published online: 22 May 2014

(C) Springer-Verlag Berlin Heidelberg 2014

\begin{abstract}
It is desirable to use computational fluid dynamics for optimization of the in-cylinder processes in low-speed two-stroke uniflow-scavenged marine diesel engines. However, the complex nature of the turbulent swirling in-cylinder flow necessitates experimental data for validation of the used turbulence models. In the present work, the flow in a dynamic scale model of a uniflowscavenged cylinder is investigated experimentally. The model has a transparent cylinder and a moving piston driven by a linear motor. The flow is investigated using phase-locked stereoscopic particle image velocimetry (PIV) and time-resolved laser Doppler anemometry (LDA). Radial profiles of the phase-locked mean and rms velocities are computed from the velocity fields recorded with PIV, and the accuracy of the obtained profiles is demonstrated by comparison with reference LDA measurements. Measurements are carried out at five axial positions for 15 different times during the engine cycle and show the temporal and spatial development of the swirling in-cylinder flow. The tangential velocity profiles in the bottom of
\end{abstract}

Electronic supplementary material The online version of this article (doi:10.1007/s00348-014-1748-y) contains supplementary material, which is available to authorized users.

K. M. Ingvorsen · K. E. Meyer · J. H. Walther $(\bowtie)$ Department of Mechanical Engineering, Technical University of Denmark, Kgs. Lyngby, Denmark e-mail: jhw@mek.dtu.dk

J. H. Walther

Computational Science and Engineering Laboratory,

ETH Zürich, Zurich, Switzerland

e-mail:walther@mavt.ethz.ch

S. Mayer

MAN Diesel \& Turbo SE, Copenhagen, Denmark

e-mail: Stefan.Mayer@man.eu the cylinder near the end of the scavenge process are characterized by a concentrated swirl resulting in wake-like axial velocity profiles and the occurrence of a vortex breakdown. After scavenge port closing, the axial velocity profiles indicate that large transient swirl-induced structures exist in the cylinder. Comparison with profiles obtained under steady-flow conditions shows that the scavenge flow cannot be assumed to be quasi-steady. The temporal development of the swirl strength is investigated by computing the angular momentum. The swirl strength shows an exponential decay from scavenge port closing to scavenge port opening corresponding to a reduction of $34 \%$, which is in good agreement with theoretical predictions.

\section{Introduction}

\subsection{Background and motivation}

Low-speed two-stroke uniflow-scavenged marine diesel engines are used to power the world's largest marine vessels such as tankers and container ships. A key process for these engines is the scavenge process (Schweitzer 1949). The scavenging is the gas exchange process where the combustion gas from the previous cycle is replaced by fresh air for the next cycle. Low-speed two-stroke marine diesel engines use the uniflow scavenging method in a configuration with scavenge ports located in the liner near the bottom of the cylinder and a single-centered exhaust valve in the cylinder head. As the piston approaches the bottom dead center (BDC), it uncovers the scavenge ports thereby starting the scavenge process. Compressed air is blown into the cylinder forcing the combustion gas out 
through the exhaust valve in a combined displacement and mixing process. The scavenge ports are cut at an angle with respect to the radial direction, typically in the range $15^{\circ}-$ $25^{\circ}$, creating a swirling in-cylinder flow. The swirl improves the scavenge process and enhances the fuel-air mixing during the combustion phase (Heywood 1988). In addition, the scavenge flow also provides cooling of the combustion chamber surfaces.

To reduce emission levels, enhance engine efficiency, and control the surface temperatures, it is desirable to use computational fluid dynamics (CFD) simulations. However, owing to their complex nature, turbulent swirling flows, such as the in-cylinder flow in uniflow-scavenged engines, are difficult to predict using CFD tools (Jakirlić et al. 2002). Experimental results are therefore needed in order to serve as validation data and to aid the development and selection of turbulence models. Despite the need for experimental data on the turbulent swirling flows in uniflow-scavenged engines, almost no works exist in the open literature presenting results suitable for validation purposes. It is this lack of experimental data, together with the need for increased understanding of the complex flow dynamics, that is the main motivation of the present work.

\subsection{Previous work}

The swirling in-cylinder flow in uniflow-scavenged engines has been the subject of earlier works. Investigations have been based on both CFD (Sung and Patterson 1982; Diwakar 1987; Uzkan 1988; Goldsborough and Blarigan 2003; Obeidat et al. 2014; Sigurdsson et al. 2014) and experiments (Percival 1955; Nakagawa et al. 1990; Sher et al. 1991; Haider et al. 2013; Ingvorsen et al. 2012, 2013). Due to the costs and limitations of experimental investigations on full-scale engines, the majority of the experimental work have been performed on simplified scale models, and often under steady-flow conditions. Ingvorsen et al. (2013) developed a simplified experimental scale model of the MAN Diesel \& Turbo 4T50ME-X research engine (Hult and Mayer 2013), which is a production-sized low-speed marine diesel engine. The flow in the model was investigated under steady-flow conditions, and the extensive measurements were performed using stereoscopic particle image velocimetry (PIV) and laser Doppler anemometry (LDA). For a $20^{\circ}$ port angle, it was found that the radial profiles of tangential velocity correspond to a concentrated vortex, with the highest velocities occurring near the cylinder axis. The axial profiles have a wake-like shape created by a velocity deficit at the axis. In the bottom of the cylinder, a central recirculation zone exists, and near the cylinder head, the vortex core is observed to precess around the exhaust valve.

\subsection{Present work}

The present work extends the work by Ingvorsen et al. (2013) by considering the flow in the engine model under dynamic conditions, where the ports are opened and closed by the piston. The main purpose of the present work is to establish a database of reliable data that can be used in the development and validation of CFD models. Secondly, the work is intended to increase the understanding of the incylinder flow field under dynamic conditions and demonstrate the differences between steady-flow and dynamic conditions.

For the investigations, a simplified scale model with a moving piston and a transparent cylinder is used (Ingvorsen et al. 2013). The design of the model ensures that the dynamics of the flow are similar to the dynamics of the flow in the full-scale engine. Due to practical limitations, compression and combustion are neglected in the model. The experiments are thus carried out under iso-thermal conditions, with both the combustion gas and scavenge air represented by ambient air at room temperature. The turbulent swirling flow in the cylinder is measured using phase-locked stereoscopic PIV and LDA.

\section{Methodology}

\subsection{Problem simplification}

In the present work, the turbulent swirling flow is investigated in a dynamic model of an engine cylinder. Dynamic model investigations are a well-established method for studying the scavenge process (Heywood and Sher 1999). They are based on the fundamental assumption that the scavenge process is only weakly dependent on the combustion process. It should be emphasized that it is not the objective of the present model to create a flow field that is identical to the flow in a full-scale fired engine. Rather, the objective of the model is to ensure that the overall dynamics of the turbulent swirling flow are the same as the flow dynamics in the engine during scavenging. Furthermore, the model must allow detailed and high-accuracy measurements to be performed. To achieve this, a series of problem simplifications are made, of which the main are:

- The compression and combustion are neglected.

- The piston motion is limited to the bottom region of the cylinder.

- The exhaust valve is fixed in the fully open position.

- The geometry is made rotational symmetric, and flat surfaces are used for the piston head, cylinder cover, and exhaust valve. 
The combustion is neglected to reduce the experimental complexity and to allow the swirling flow to be studied in an isolated manner. The main discrepancy arising from this simplification is related to the fluid inertia at the time of scavenge port opening. In the case with combustion, the gas will blow out through the exhaust valve due to the increased in-cylinder pressure (the blowdown process). In contrast, the flow in the model will move into the cylinder due to the downward motion of the piston. The difference in fluid inertia results in a phase lag in the scavenge process and a higher effective flow resistance in the model compared to the engine. Additional effects of the missing combustion include neglecting a contribution to the angular momentum from the fuel injection and neglecting density differences between the gas in the cylinder and the scavenge air.

The piston stroke is limited to ensure a high-quality optical access by avoiding scratching and depositing of seeding liquid on the transparent cylinder wall. The limited stroke and fixed exhaust valve imply that the compression is neglected. It is shown that compression only has a minor effect on the scavenge process when running under motored conditions (Joenson 2011). Furthermore, the combustion chamber in the real engine is approximately diskshaped, and the compression does therefore not result in any significant swirl modification (Heywood 1988).

Finally, it should be noted that although the stroke is limited to the bottom region of the cylinder, the piston motion during the scavenge period is reproduced with a good accuracy, both in terms of position, velocity, and acceleration.

\subsection{Problem scaling}

To ensure that the flow dynamics in the experimental scale model are similar to the dynamics in a full-scale engine, a short analysis is carried out considering the main parameters characterizing the scavenge flow in a uniflow-scavenged engine. For the analysis, a characteristic scavenge velocity is introduced as $U_{\mathrm{sc}}=m_{\mathrm{sc}}\left(\rho t_{\mathrm{sc}} A_{\mathrm{cyl}}\right)^{-1}$, where $m_{\mathrm{sc}}$ is the mass of scavenged gas per cycle, $\rho$ is the gas density, $A_{\text {cyl }}$ is the cross-sectional area of the cylinder, and $t_{\mathrm{sc}}$ is the scavenge time. In the present work, the scavenge time refers to the time from scavenge port opening $\left(t_{\mathrm{SPO}}\right)$ to scavenge port closing $\left(t_{\mathrm{SPC}}\right)$ such that $t_{\mathrm{sc}}=t_{\mathrm{SPC}}-t_{\mathrm{SPO}}$. Using the scavenge velocity, the flow dynamics can be characterized by the following three nondimensional quantities: (1) the Reynolds number $R e=U_{\mathrm{sc}} D / v$, where $D$ is the cylinder diameter and $v$ is the kinematic viscosity of the gas, (2) the nondimensional scavenge velocity $U_{\mathrm{sc}}^{*}=U_{\mathrm{sc}} t_{\mathrm{sc}} / D$, and (3) the nondimensional scavenge time $t_{\mathrm{sc}}^{*}=t_{\mathrm{sc}} / t_{\mathrm{cyc}}$, where $t_{\mathrm{cyc}}$ is the period of the engine cycle.
The nondimensional scavenge velocity $U_{\mathrm{sc}}^{*}$ is directly related to both the delivery ratio $\Lambda$ and the engine Strouhal number $S t$. The delivery ratio is given by $\Lambda=m_{\mathrm{sc}} / m_{\mathrm{ref}}$, where $m_{\text {ref }}$ is the mass of air necessary to fill the cylinder at scavenge box conditions. The engine Strouhal number, given by $S t=n D / U_{\text {sc }}$, where $n$ is the engine speed, is an important similarity parameter when modeling the scavenge process (Blair 1996). A fourth nondimensional parameter, the Euler number, is sometimes also used. The Euler number $E u=\Delta P /\left(\frac{1}{2} \rho U_{\mathrm{sc}}^{2}\right)$ is the nondimensional scavenge pressure and expresses the effective flow resistance through the cylinder. However, for the present investigations, the Euler number is not important as it has negligible impact on the flow dynamics and only indicates if the required pumping power is the same for the model and the engine.

To ensure similarity of the flow dynamics, the three nondimensional parameters should ideally be identical for the experimental model and the full-scale engine. The nondimensional parameters for the 4T50ME-X research engine are $R e=2,600,000, U_{\mathrm{sc}}^{*}=5.2$, and $t_{\mathrm{sc}}^{*}=0.21$ (Sigurdsson et al. 2014). It is possible to match the nondimensional velocity and time, but due to practical limitations, it is not possible to match the large Reynolds number. It is, however, known that the flow becomes approximately independent of the Reynolds number for sufficiently large Reynolds numbers. In the steady-flow investigations, it is shown that both the mean and rms velocities become independent of the Reynolds number for $R e \gtrsim 50,000$ (Ingvorsen et al. 2013). The required scavenge velocity and scavenge time for the experimental model can therefore be determined from the model diameter $D$ and requiring that the model Reynolds number is $R e \gtrsim 50,000$.

\subsection{Flow rig}

A schematic of the experimental model is presented in Fig. 1, and a detailed description of the experimental setup is given in Ingvorsen et al. (2013). In short, the model consist of a transparent glass cylinder with an internal diameter of $D=190 \mathrm{~mm}$. The flow enters the cylinder through a port section placed in the bottom of the glass cylinder. The total length of the cylinder, measured from the bottom of the ports to the cylinder head, is 5.00D. The port section can be changed making it possible to test different port configurations with, for example, different port angles and shapes, and different number of ports. The port configuration used for the present work is shown in Fig. 1b. It has 30 equally spaced ports with port angles of $\alpha=20^{\circ}$ and a port height of $0.40 D$. Two inlet plates are used to guide the ambient air from the surroundings to the ports. The flow exits the cylinder through an exhaust pipe 

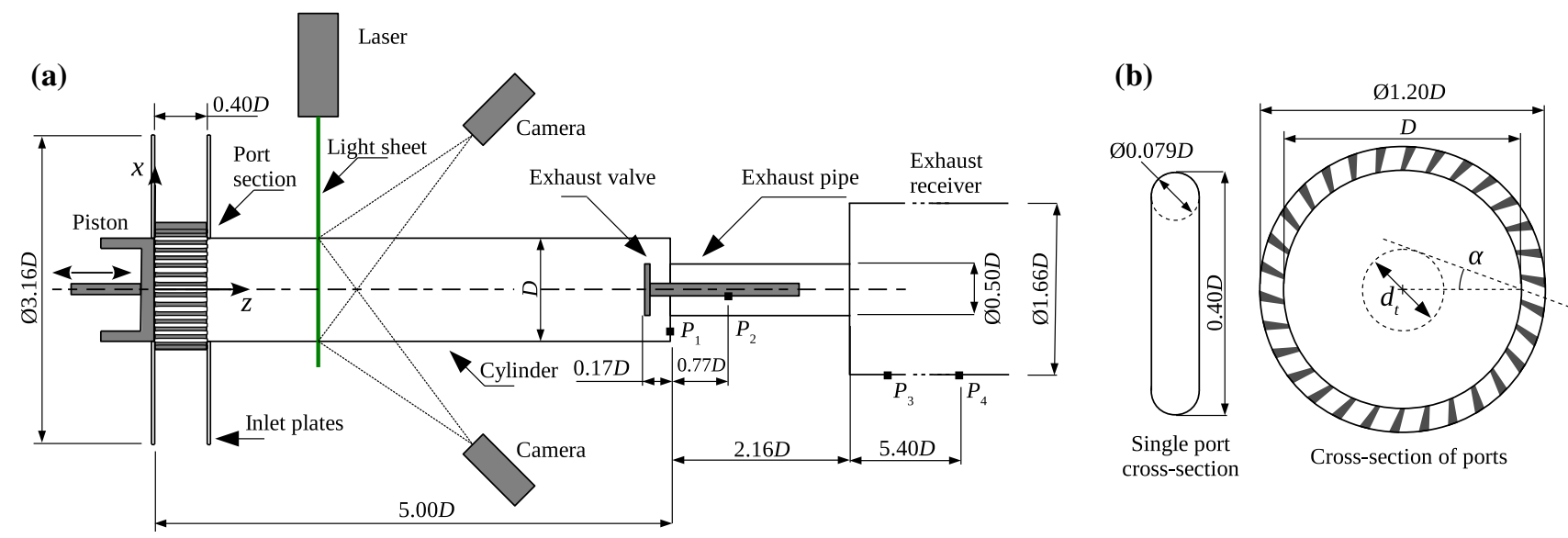

Fig. 1 a Sketch of the model and optical setup. b Details of the port section geometry. Adapted from Ingvorsen et al. (2013)

with a diameter of $0.50 \mathrm{D}$. To enter the exhaust pipe, the flow must go around the static exhaust valve. From the exhaust pipe, the flow enters the exhaust receiver, which is a large cylindrical volume with a diameter of $1.66 \mathrm{D}$ and length of $6.77 D$. The exhaust receiver is connected to a frequency controlled centrifugal fan by a contraction. The fan drives the flow through the model and exhausts directly into the ambient air. Both the cylinder head, the exhaust valve, and the piston surface are flat. The gap between the piston side and cylinder wall is $0.005 D$ wide and is sealed by two custom-made piston rings. The piston rings are made in Teflon to reduce friction and limit abrasion of the glass wall. The axial distance from the piston surface to the contact point between the upper piston ring and the cylinder wall is $0.03 D$. Therefore, the effective port opening occurs slightly before the piston surface moves below the top of the scavenge ports. A Cartesian coordinate system is defined as shown in Fig. 1a. The $z$-axis is coincident with the cylinder axis, and the origin is at the axial position corresponding to the bottom of the ports. Note that also a cylindrical coordinate system is used later in the paper, when presenting the PIV results.

The piston motion is controlled by a linear motor (LinMot P01-37Sx120F-HP) and a programmable servo controller. At the cycle start, the piston is located with the piston surface at $z / D=1.00$, and it then moves to $z / D=0.00$, corresponding to $100 \%$ open ports, and back to $z / D=1.00$ in a sinusoidal motion. Having returned to $z / D=1.00$, the piston has a standstill period before the cycle is repeated. The standstill period is included to obtain the correct nondimensional scavenge time $t_{\mathrm{sc}}^{*}$ without performing the full stroke. Matching the nondimensional scavenge time is important in order to give the swirl sufficient time to decay before the next cycle. The total cycle time is $t_{\mathrm{cyc}}=1.20 \mathrm{~s}$ and both the motion and standstill period are $0.50 t_{\mathrm{cyc}}$ in duration. Defining the time of port

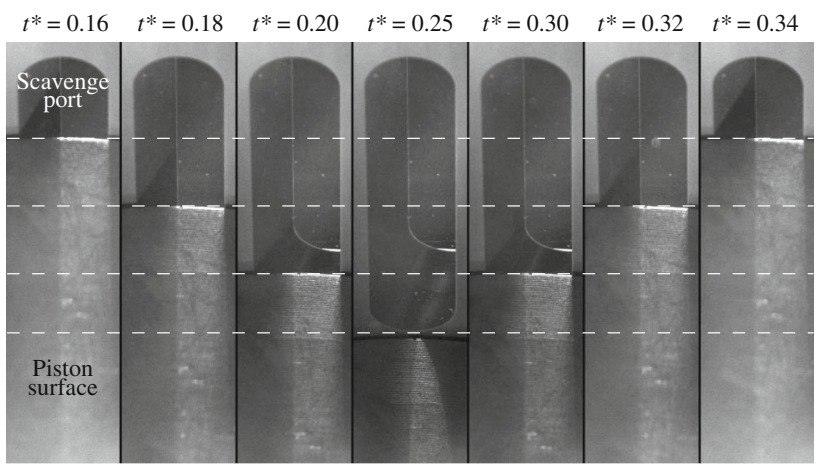

Fig. 2 Close-ups of the piston surface and a scavenge port at different times $t^{*}=t / t_{\text {cyc }}$ acquired with the PIV system

opening and port closing as the times when the piston surface is at $z / D=0.40 D$, the port opening and closing occur at $t_{\mathrm{SPO}} / t_{\mathrm{cyc}}=0.14$ and $t_{\mathrm{SPC}} / t_{\mathrm{cyc}}=0.36$, respectively. To relate the velocity and pressure measurements to the piston motion, a trigger signal is sent from the servo controller at port opening.

The piston motion is investigated by sampling the trigger signal and by taking photographs of the position of the piston near the ports. Analysis of the trigger signal shows that the variations in the cycle period $t_{\mathrm{cyc}}$ are less than $1.0 \mathrm{~ms}$. To investigate the accuracy of the instantaneous piston position, the PIV system is used to acquire images of the piston at different port openings. Close-ups of the images, showing the piston and a single port, are presented in Fig. 2. The close-ups show that the instantaneous piston position is symmetric around the bottom dead center and in good agreement with the specified motion. The cycle-tocycle variations in the piston position are small, being typically less than $0.5 \mathrm{~mm}$.

The fan speed is adjusted such that the Reynolds number is $R e=U_{\mathrm{sc}} D / v=50,000$, which for standard ambient 
conditions $\left(20^{\circ} \mathrm{C}, 1.0 \mathrm{~atm}\right)$ corresponds to $U_{\mathrm{sc}}=3.96 \mathrm{~m} / \mathrm{s}$. The nondimensional scavenge velocity and scavenge time for the model thus become $U_{\mathrm{sc}}^{*}=5.4$ and $t_{\mathrm{sc}}^{*}=0.22$, respectively. These are within $5 \%$ of the corresponding numbers for the full-scale engine. It can therefore be expected that the flow physics in the model and in the engine is similar. It should be noted that when adjusting the fan speed, the scavenge velocity is estimated from timeresolved measurements of the pressure difference over the valve using a pressure drop coefficient determined under steady-flow conditions (see Sect. 2.4). The scavenge velocity based on the pressure difference is not sufficiently accurate for normalization purposes. Instead, the bulk velocity at $t / t_{\mathrm{cyc}}=0.25$, corresponding to fully open ports, is used. The bulk velocity is found to be $V_{b}=4.70 \mathrm{~m} / \mathrm{s}$ based on the radial profile of axial velocity measured with PIV at $z / D=1.50$.

For CFD simulations of the flow in the model, the following recommendations are given concerning the inlet and outlet boundaries. The inlet boundary should be located some distance upstream the entrance to the inlet plates, and a pressure condition should be employed. At this location, the flow can be assumed to be laminar, and at standard ambient conditions $\left(20^{\circ} \mathrm{C}, 1.0 \mathrm{~atm}\right)$. The outlet boundary should be the exhaust receiver cross section at the axial position of pressure tab $P_{4}$, see Fig. 1a. The pressure distribution over the cross section can be assumed uniform, and the measured phase-averaged pressure can therefore be used as the boundary condition (cf. Ingvorsen et al. 2013).

\subsection{Pressure measurements}

The pressure in the model is measured using four-wall pressure tabs. The pressure tabs are located in the cylinder head, on the exhaust valve rod, and on the side of the exhaust receiver $0.37 \mathrm{D}$ and $5.40 \mathrm{D}$ downstream the inlet to the exhaust receiver (cf. Fig. 1a). The pressure is sampled at $5.0 \mathrm{kHz}$ using four pressure transducers and a LabVIEW acquisition system. The pressure is recorded for 50 consecutive cycles, and the phase-averaged pressure is calculated. The statistical uncertainty on the phase-averaged pressure is estimated to be $\pm 0.56 \frac{1}{2} \rho V_{b}^{2}$, corresponding to approximately $\pm 1 \%$ of the pressure difference over the model when the piston is at $\operatorname{BDC}\left(P_{4}\left(t / t_{\mathrm{cyc}}=0.25\right)\right)$.

\subsection{Laser Doppler anemometry (LDA)}

The details of the laser Doppler anemometry (LDA) system are given in Ingvorsen et al. (2013). To avoid the laser beams being deflected by the curved glass wall, the LDA optics are oriented such that both beams are located in the horizontal plane that intersects the cylinder axis (the $x, z-$ plane). The LDA system is therefore limited to measuring the axial velocity component. The system is operated in burst mode which results in velocity measurements randomly distributed in time. In order to estimate the phaseaveraged velocity, time series corresponding to 50 cycles are acquired for each measurement point, and a temporal binning is carried out using a window size of $\Delta t / t_{\mathrm{cyc}}=0.001$. The resulting statistical uncertainty on the phase-averaged velocities is estimated to be $2 \%$ of $V_{b}$. The seeding used for the measurements is di-ethyl-hexyl-sebacate (DEHS) droplets with diameters in the range 0.1$2.0 \mu \mathrm{m}$ generate by a Laskin nozzle.

\subsection{Stereoscopic particle image velocimetry (PIV)}

\subsubsection{PIV system}

Stereoscopic particle image velocimetry (PIV) is the primary measurement method used in the present study, and the measurements are performed with a commercial PIV system from Dantec Dynamics. The laser is a $200 \mathrm{~mJ}$ double cavity $\mathrm{Nd}$ :YAG laser. The light sheet thickness in the field of view is $2-3 \mathrm{~mm}$ and the laser is operated at full power for the measurements. The cameras are 12 bit CCD cameras (Dantec Dynamics HiSense Mk II) with a chip size of $1,344 \times$ 1,024 pixels and a pixel pitch of $6.45 \mu \mathrm{m}$. The cameras are fitted with $60 \mathrm{~mm}$ lenses using Scheimpflug mounts, and the F-number is set to 11 for both cameras. A schematic of the PIV setup is included in Fig. 1a. The laser is positioned next to the model, such that the light sheet illuminates the cylinder cross section. The cameras are positioned at the downstream side of the light sheet with one camera on the same side as the laser (backward scatter position) and one camera on the opposite side of the cylinder (forward scatter position). The laser and cameras are mounted on a traverse aligned with the cylinder axis allowing measurements to be performed at different axial positions without recalibrating. Measurements are performed at the five axial positions given by $z / D=\{1.00,1.50,2.00,3.00,4.00\}$.

The image acquisition, camera calibration, and PIV analysis are carried out using the DynamicStudio version 3.3 software. A third-order polynomial image mapping approach is used for the stereo viewing calibration. This approach is chosen as it can account for the nonlinear image distortions created by the curved glass wall. To enhance the accuracy of the calibration, a disparity correction is performed. Before analyzing the particle images, background images are subtracted to enhance the image quality. The background images are obtained by calculating the minimum images (in terms of light intensity) of their respective image series. The particle images are processed using a three-step adaptive correlation algorithm 
Table 1 Measurement times for the PIV measurements together with the piston positions and port opening ratios

\begin{tabular}{lll}
\hline$t / t_{\text {cyc }}$ & $z / D$ & Port opening \\
\hline 0.00 & 1.00 & - \\
0.10 & 0.65 & - \\
0.16 & 0.30 & $25 \%$ open \\
0.18 & 0.20 & $50 \%$ open \\
0.20 & 0.10 & $75 \%$ open \\
0.25 & 0.00 & $100 \%$ open \\
0.30 & 0.10 & $75 \%$ open \\
0.32 & 0.20 & $50 \%$ open \\
0.34 & 0.30 & $25 \%$ open \\
0.40 & 0.65 & - \\
0.50 & 1.00 & - \\
0.60 & 1.00 & - \\
0.70 & 1.00 & - \\
0.80 & 1.00 & - \\
0.90 & 1.00 & - \\
\hline
\end{tabular}

with a final interrogation area size of $32 \times 32$ pixels and $50 \%$ overlap. Spurious vectors are detected using a local median criterion and replaced by interpolation.

To obtain phase-locked measurements, the timing of the laser and cameras is controlled by the trigger signal from the servo controller. By specifying a trigger delay in the software, it is possible to measure at arbitrary times during the cycle. Measurements are performed at 15 different times during the cycle. The measurement times are presented in Table 1 together with the corresponding piston positions, and port opening ratios.

\subsubsection{Post processing}

For each measurement series, corresponding to a given time and axial position, the three-component instantaneous velocity field $v_{i}, i=1,2,3$ is recorded for 100 consecutive cycles. The phase-locked mean velocity field $V_{i}$ and the rms velocity field $V_{i, \text { rms }}$ are obtained by computing the ensemble average and the standard deviation of the measurement series. An example of the phase-locked mean velocity field is shown in Fig. 3. The field of view covers an approximately rectangular region with the left corners close to the cylinder wall. The phase-locked mean and rms velocity fields are found to be approximately axisymmetric. It is therefore convenient to represent them using a cylindrical coordinate system with coordinates $r, \theta, z$ and corresponding velocity components $V_{r}, V_{\theta}, V_{z}$. The mean and rms velocity fields are converted to radial profiles by averaging in the $\theta$-direction which in practice is done by performing a radial binning (see Ingvorsen et al. 2013 for details).

Due to the inevitable inaccuracies of the experimental setup, the swirl center of the mean flow field will in general

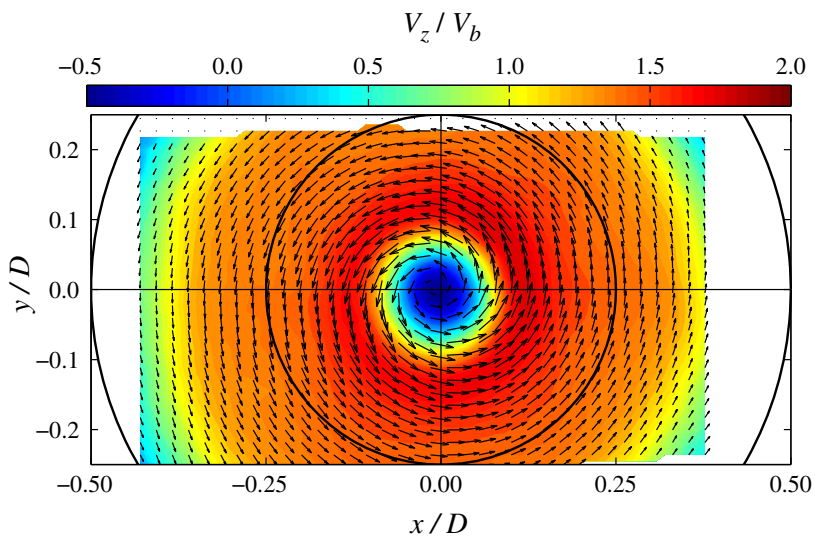

Fig. 3 Phase-locked mean velocity field at $z / D=1.00$ and $t / t_{\text {cyc }}=0.30$. The in-plane components are shown by vectors (every second vector in each direction is shown), and the axial component $V_{z} / V_{b}$ is shown by color

not coincide exactly with the cylinder center. This phenomenon is most pronounced for measurements taken shortly after the port opening, where the flow is subjected to large changes without having been stabilized by the strong swirl present later in the cycle. To account for the discrepancies between the mean swirl centers and geometrical center, the radial profiles in the range $r / D=0.00-0.30$ are generated using the mean swirl center as the origin for the binning, whereas the profiles in the range $r / D=0.30-0.50$ are generated using the geometrical center as origin.

\subsubsection{Estimation of accuracy of measured velocity profiles}

The accuracy of the radial velocity profiles, obtained from the PIV measurements, is evaluated both by comparing profiles from repeated measurement series, and by comparing the PIV profiles with profiles measured with LDA.

Repeated measurements are carried out at $z / D=1.00$ for $t / t_{\text {cyc }}=0.25$ and $t / t_{\text {cyc }}=0.30$. The comparison of the profiles is presented in Fig. 4 and shows a high repeatability. The mean discrepancy between the profiles is found to be $3 \%$ of $V_{b}$. It is noted that the largest discrepancies are observed for the axial velocity component near the center and close to the wall. One reason for this is that the profiles in these regions are based on a smaller amount of data due to the combination of the radial binning and the rectangular field of view. It is further noted that the agreement of the profiles at $t / t_{\text {cyc }}=0.30$ is higher than at $t / t_{\text {cyc }}=0.25$ and indicates that the increase in swirl strength has stabilized the flow making the measurements more repeatable.

The axial velocity profiles obtained by PIV can be compared to the LDA measurements. In this case, the comparison is made for all axial positions and measurement times. Examples of the comparison are shown in 
Fig. 5 for the times $t / t_{\mathrm{cyc}}=0.25$ and $t / t_{\mathrm{cyc}}=0.30$. The comparison shows a good agreement between the PIV and LDA profiles with a mean discrepancy of $6 \%$ of $V_{b}$. It
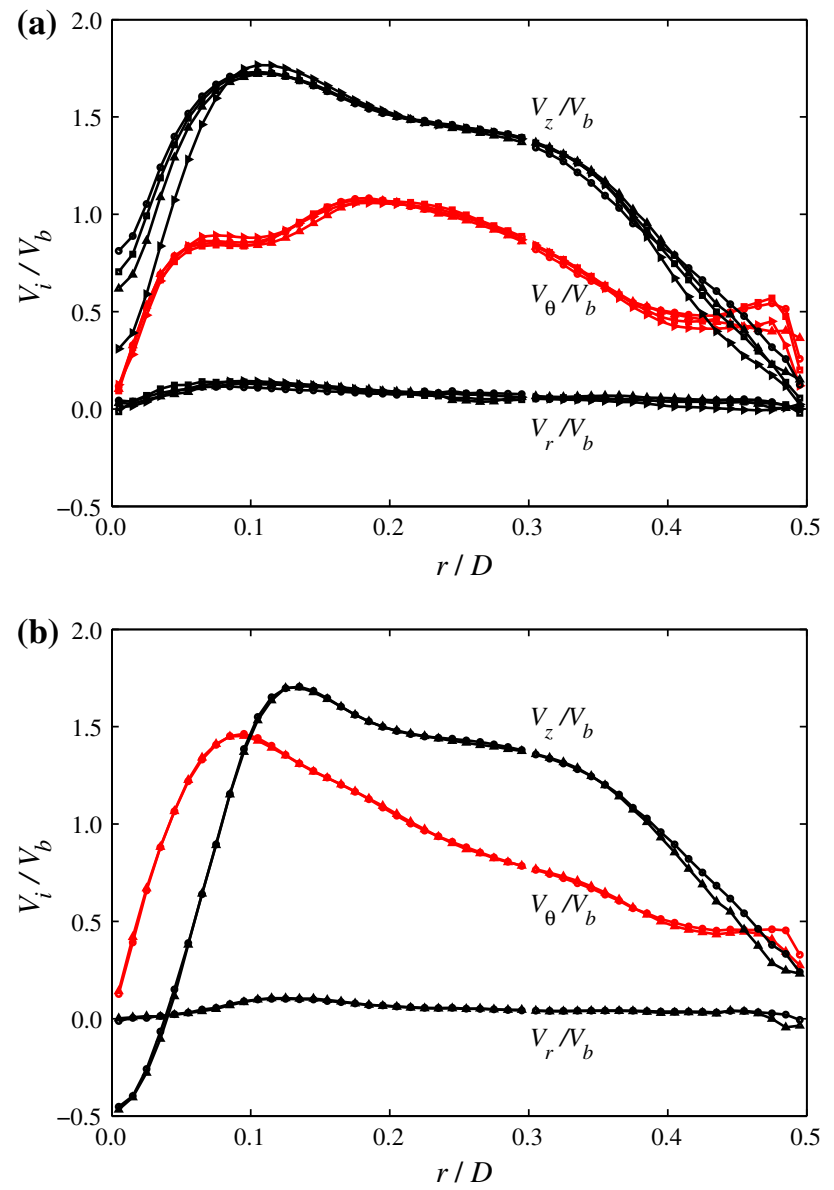

Fig. 4 Comparison of repeated PIV measurements at $z / D=1.00$ for two different times. a $t / t_{\mathrm{cyc}}=0.25 ; \mathbf{b} t / t_{\mathrm{cyc}}=0.30$. Symbols denote the different measurement series should, however, be emphasized that this discrepancy is not only the result of the PIV uncertainty, but also accounts for the uncertainties in the LDA measurements.

\section{Results}

\subsection{Pressure}

The phase-averaged pressure at the cylinder head $P_{1}$ and at the exhaust receiver outlet $P_{4}$ is presented in Fig. 6. At the scavenge port opening, a rapid increase is observed at both locations. The pressure at the cylinder head continues to increase reaching an approximately constant value at $t / t_{\mathrm{cyc}}=0.23$. During the scavenging, fast oscillations are observed in the cylinder pressure. The frequency of the oscillations is $f=c /(4 L)$, where $c$ is the speed of sound and $L$ is the cylinder length. This is the fundamental frequency of a cylinder with one end open and one end closed (Young and Freedman 2004). This shows that the bottom end of the cylinder acts as an open end when the ports are uncovered, whereas the top end acts as closed even though the exhaust valve is open. This behavior is consistent with oscillations observed in measurements and simulations of a full-scale fired engine (Sigurdsson et al. 2014).

After the initial increase following the port opening, the pressure at the exhaust receiver outlet $P_{4}$ is reduced again, reaching an approximately constant value of $P /\left(\frac{1}{2} \rho V_{b}^{2}\right)=$ 42 at $t / t_{\mathrm{cyc}}=0.23$. At scavenge port closing (SPC), both pressures show a rapid reduction resulting in a new series of large oscillations. The oscillations after port closure are significantly slower than the in-cylinder oscillations observed under scavenging. These slow oscillations are expected to be the result of a Helmholtz resonator
Fig. 5 Comparison of radial profiles of axial velocity measured with PIV (solid lines) and LDA (open circles). a $t / t_{\mathrm{cyc}}=0.25 ; \mathbf{b} t / t_{\mathrm{cyc}}=0.30$
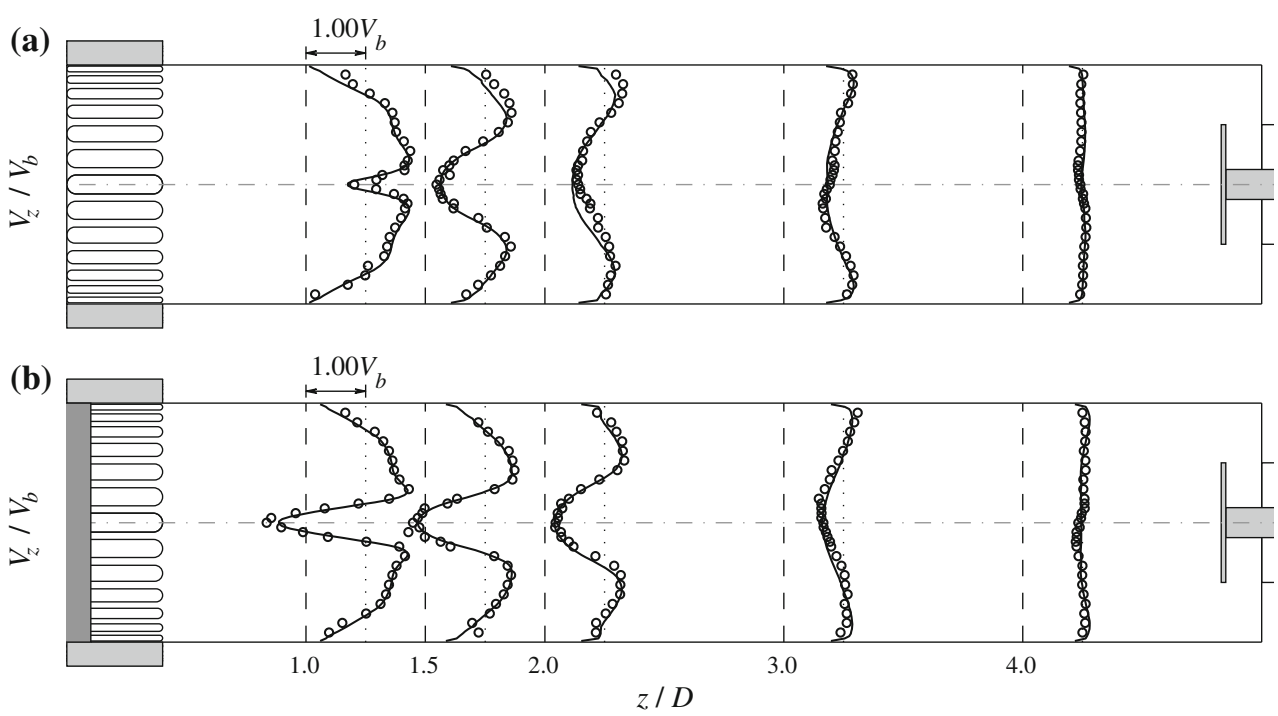


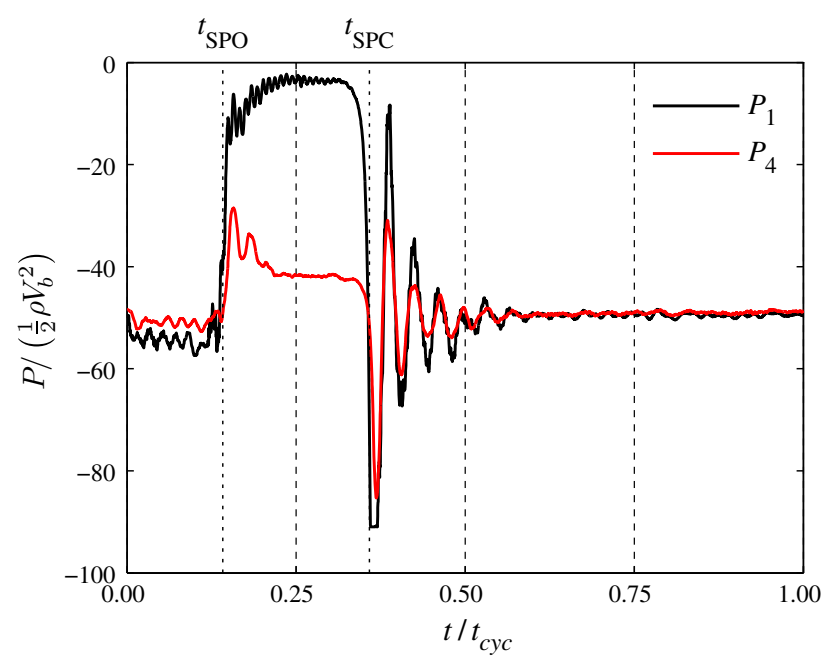

Fig. 6 Phase-averaged pressure as a function of time, measured at the cylinder head $\left(P_{1}\right)$ and at the exhaust receiver outlet $\left(P_{4}\right)$

phenomenon, where the cylinder and exhaust receiver volumes act as springs and the exhaust pipe volume acts as a mass. The oscillations are quickly damped out, and the pressure becomes approximately constant at $t / t_{\mathrm{cyc}}=0.60$.

The nondimensional pressure difference over the scavenge ports and exhaust valve can be estimated as $\Delta P_{\text {ports }}=$ $P_{1} / P_{\text {ref }}$ and $\Delta P_{\text {valve }}=\left(P_{2}-P_{1}\right) / P_{\text {ref }}$, respectively, where $P_{\text {ref }}=\frac{1}{2} \rho V_{b}^{2}$. When the piston is at BDC, corresponding to $t / t_{\text {cyc }}=0.25$, the pressure differences are $\Delta P_{\text {ports }}=-3.7$ and $\Delta P_{\text {valve }}=-45.6$, showing that the valve is the main contribution to the total pressure loss.

\subsection{Flow rate}

The phase-averaged flow rate $Q^{\prime}$ through the cylinder during the period with piston motion $\left(t / t_{\text {cyc }}=0.00-0.50\right)$ is shown in Fig. 7. The flow rate is estimated by integrating the radial profiles of axial velocity obtained from the PIV and LDA measurements at $z / D=1.50$. The flow rate estimated from the pressure difference over the exhaust valve is also included. It is expected that the flow rate estimated from the PIV profiles is the most accurate; however, the estimates based on the LDA and pressure measurements are time-resolved, and therefore give a more complete description of the flow development. In general, good agreement is observed between the PIV and LDA estimates, whereas the estimates based on the pressure difference over the exhaust valve show a lag after port opening and a lower maximum flow rate. The larger uncertainties on the estimates based on the pressure difference, compared to the PIV and LDA estimates, are expected as the used pressure drop coefficient was obtained under steady-flow conditions.

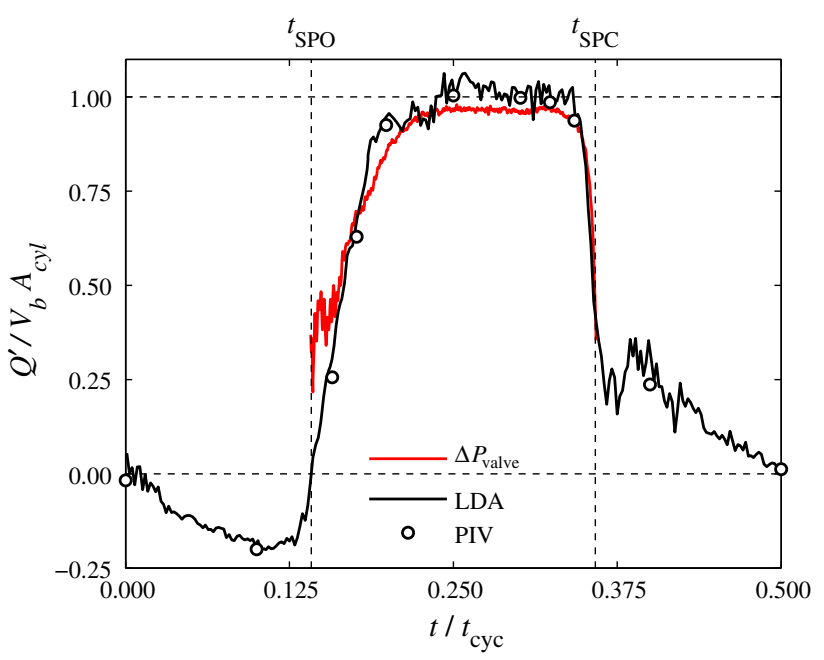

Fig. 7 Phase-averaged flow rate during piston motion, estimated from the PIV and LDA profiles and the pressure difference over the exhaust valve $\left(\Delta P_{\text {valve }}\right)$

From the cycle start to the scavenge port opening, the flow rate is negative due to the downward motion of the piston. After port opening, the flow direction changes and the flow rate increases to an approximately constant value shortly before the piston reaches $z / D=0.00$. The flow rate remains approximately constant until the ports are almost completely covered, at which point it shows a rapid decrease. The relatively slow change in flow rate at port opening compared to port closing is the result of the fluid inertia. At the time of port opening, the air is being pulled into the cylinder, through the exhaust valve, by the piston motion. The flow direction therefore has to change before the scavenging can commence. This is different to the process in a fired engine, where the gas is moving out of the cylinder before port opening due to the blowdown process.

\subsection{Phase-averaged velocity profiles}

The radial profiles of phase-averaged radial $V_{r}$, tangential $V_{\theta}$, and axial velocity $V_{z}$ are presented in Figs. 8, 9, and 10 for different times during the cycle. To aid the interpretation of the results, the profiles are plotted in the model geometry, where also the piston position is shown. The $V_{r}$ and $V_{z}$-profiles are mirrored in the cylinder axis, and the $V_{\theta}$-profiles are mirrored in both the cylinder axis and the datum lines. Please note that the full data set is presented in Online Resource 1, where the profiles are plotted in both the model geometry but also in an $x, y$-plot format, facilitating accurate reading of the profiles.

The radial velocities are in general much smaller than the tangential and axial velocities, and they are furthermore approximately zero during the period when the ports 


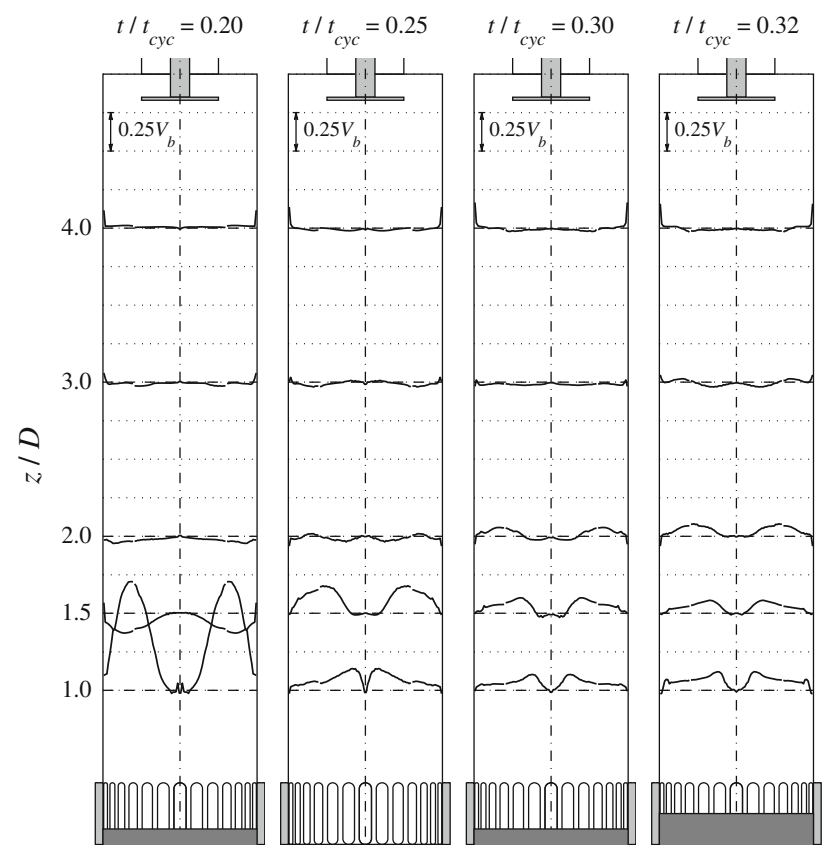

Fig. 8 Radial profiles of the phase-averaged radial velocity $V_{r}(r)$ for selected times in the port open period

are closed. The radial profiles are therefore only presented at four selected times during the port open period (Fig. 8). From the plots, it is seen that significant nonzero velocities only appear in the bottom half of the cylinder. At $t / t_{\mathrm{cyc}}=0.20$ it is observed that the flow is diverging at $z / D=1.0$ (positive $V_{r}$ ) and converging at $z / D=1.5$ (negative $V_{r}$ ), corresponding to the flow going around an obstacle. For the later times, only the diverging profile is pronounced, and it is observed also for the higher $z / D$ values. The diverging, or outward, motion of the flow is created by a region of low axial velocity in the center of the cylinder. As the scavenging progresses, the region with low axial velocity gets larger due to the increased swirl, why the flow shows the diverging behavior at higher $z / D$ values.

Considering the development of the tangential velocity (Fig. 9), the following is noted. At the cycle start $\left(t / t_{\text {cyc }}=0.00\right)$, a pre-swirl or residual swirl exist and the swirl strength is approximately the same for the different axial positions. The profiles consist of a central region where the velocity increases with radial position, an annular region where the velocity is approximately constant or slightly decreasing, and a thin wall boundary layer where the velocity shows a rapid decrease. For $t / t_{\mathrm{cyc}}=0.10$ and $t / t_{\mathrm{cyc}}=0.16$, the profiles show no or limited effect of the downward motion of the piston and the port opening. At $t / t_{\mathrm{cyc}}=0.18$, an increase in the tangential velocity near the wall is observed for the $z / D=$ 1.00 profile, showing that the scavenge air has now penetrated to this axial position. At $t / t_{\mathrm{cyc}}=0.20$, the tangential velocity at $z / D=1.00$ is significantly increased and the position of the maximum velocity has moved closer to the wall and is now located at approximately $r / D=0.30$. The introduction of swirl can now also be observed at the $z / D=1.50$ profile. At $t / t_{\mathrm{cyc}}=0.25$, corresponding to fully open ports, the maximum tangential velocity is further increased and the profile shape becomes complex with three positions with local maximum velocity. The profiles at $z / D=1.50$ and $z / D=2.0$ are also significantly affected, showing approximately solid body rotation in the central region with maximum velocities at approximately $r / D=0.30$.

At $t / t_{\text {cyc }}=0.30$, corresponding to $25 \%$ closed ports, the tangential profile at $z / D=1.00$ has the maximum velocity close to the center axis, with the highest observed tangential velocity for the entire cycle. The tangential velocity profile now consists of a small central region with a high gradient and an approximately solid body rotation, and an annular region where the velocity decreases as a function of the radial position. The profile shape is relatively smooth and corresponds to a concentrated vortex (Steenbergen and Voskamp 1998), which is similar to the profiles found in the steady-flow case (Ingvorsen et al. 2013). The profiles near the cylinder head have not attained this profile shape and still have the maximum velocity close to the wall. At $t / t_{\mathrm{cyc}}=0.32$, the ports are $50 \%$ closed, and the maximum swirl velocity is reduced at the first two axial positions, whereas it has increased slightly at the downstream positions. The tangential velocity profiles show nonmonotonic behavior near the cylinder wall. At $t / t_{\mathrm{cyc}}=0.34$, the ports are $75 \%$ closed and the tangential profile at $z / D=1.00$ have become highly complex with multiple local maximum locations. The profiles for $z / D=1.50$ and $z / D=2.00$ are less affected and show a small reduction in maximum tangential velocity, while the profiles for $z / D=3.00$ and 4.00 show a small increase. The tangential velocity for the $z / D=4.00$ position is approximately constant, except close to the center and the wall. At $t / t_{\mathrm{cyc}}=0.40$, the scavenge ports have closed, and a large change in velocity profiles is observed for the first three positions. They become similar to the downstream profiles where the central region has an approximately solid body rotation, and the annular region has an approximately constant velocity. At $t / t_{\text {cyc }}=0.50$, the piston has returned to $z / D=1.00$, and the profiles at $z / D=1.50$ and 2.00 show approximately solid body rotation over the majority of the cross section with only a thin boundary layer on the wall. In the remaining standstill period $\left(t / t_{\mathrm{cyc}}=0.60-1.00\right)$, the profiles in general become more similar for the different axial positions, showing a rapid increase from the center followed by an annular region with approximately constant velocity. 


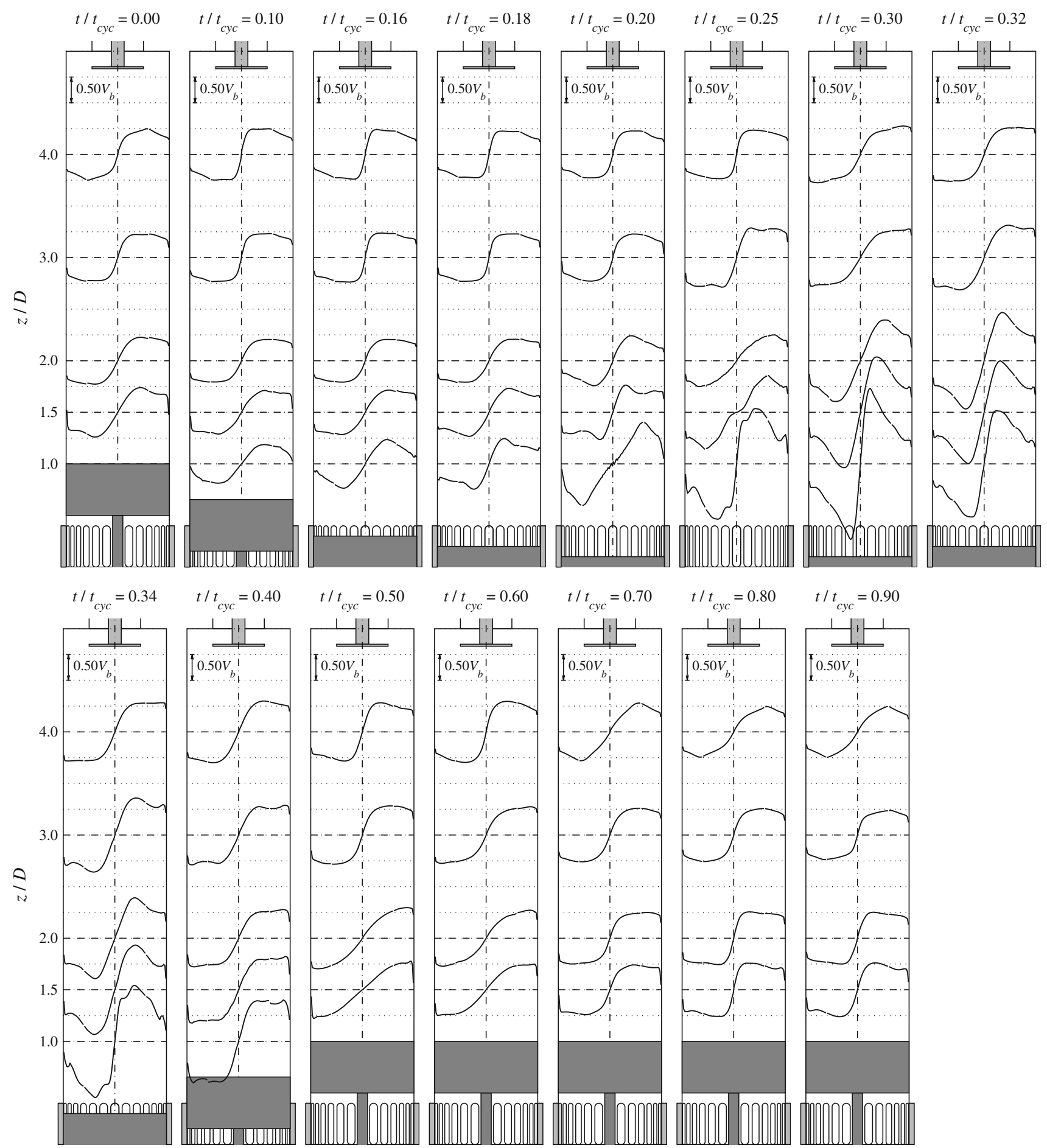

Fig. 9 Radial profiles of the phase-averaged tangential velocity $V_{\theta} / V_{b}$ for the different times during the cycle

Considering the radial profiles of axial velocity (Fig. 10), it is seen that at the cycle start $t / t_{\mathrm{cyc}}=0.00$, negative axial velocities exist near the cylinder axis for all axial positions. It is expected that the nonzero axial velocities are caused by the residual swirl. At $t / t_{\mathrm{cyc}}=0.10$, negative velocities are observed over the entire cross section for all axial positions showing that the air is pulled down by the piston. At $t / t_{\mathrm{cyc}}=0.16$, the flow direction has changed and positive axial velocities are observed for all axial positions. The profile at $z / D=1.00$ has a small peak near the wall showing that air is blown into the cylinder in an annular region close to the liner. The profiles for $z / D=$ 1.50 and 2.00 are approximately constant, whereas the profiles at $z / D=3.00$ and 4.00 show a peak near the 


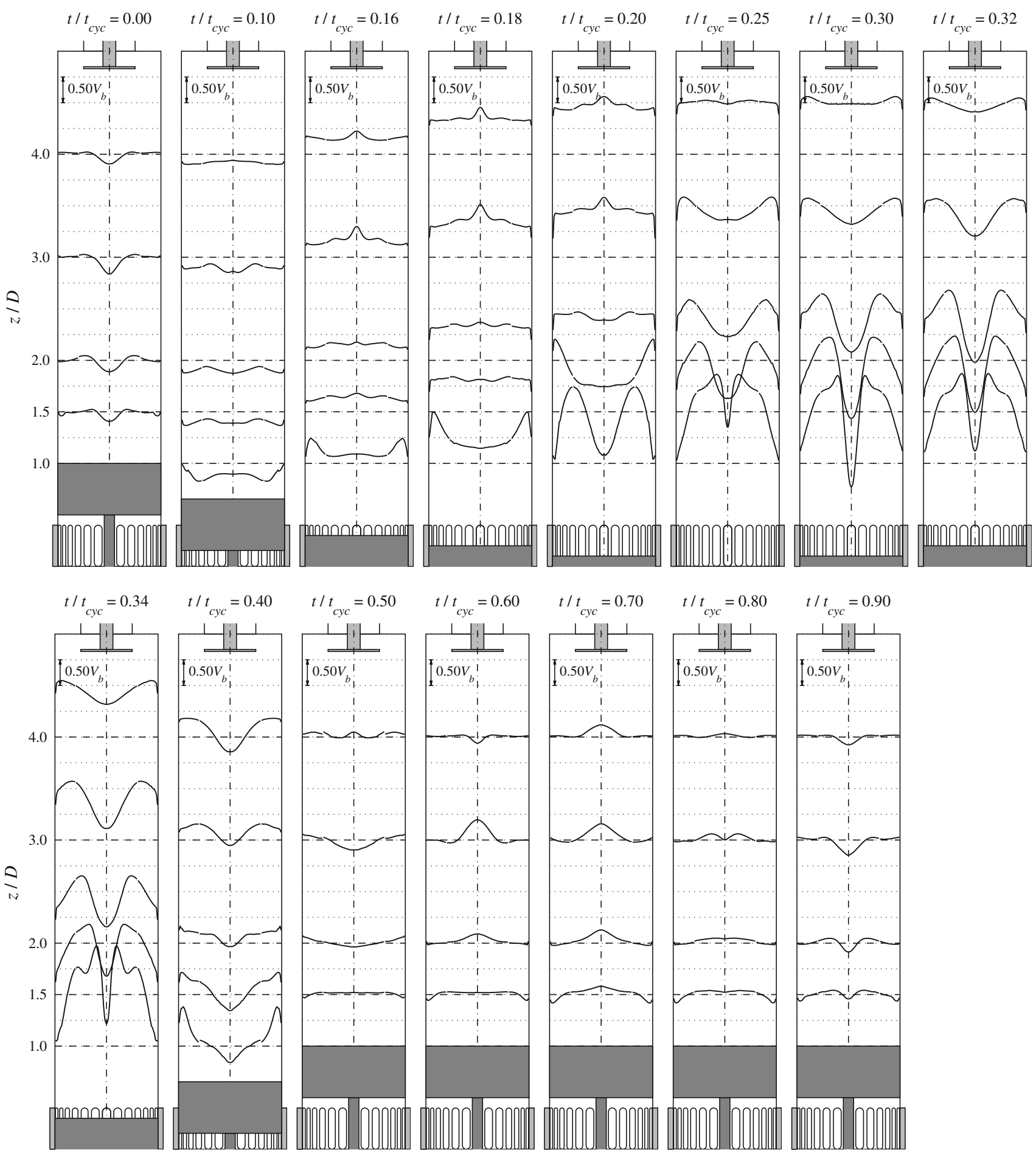

Fig. 10 Radial profiles of the phase-averaged axial velocity $V_{z} / V_{b}$ for the different times during the cycle

cylinder axis. The peak is expected to be caused by a suction effect along the swirl center. A similar effect was observed for the steady-flow case (Ingvorsen et al. 2013). At $t / t_{\mathrm{cyc}}=0.18$, the general trend is unchanged, but the magnitude of the velocities has increased. The shape of the $z / D=1.00$ axial profile is wake-like with a wide deficit.
At $t / t_{\mathrm{cyc}}=0.20$, the wake-like shape becomes more pronounced with higher axial velocities and a more narrow deficit. The profile at $z / D=1.50$ has now also developed a wake-like shape with a wide deficit. At $t / t_{\mathrm{cyc}}=0.25$ all profiles except the $z / D=4.00$ profile have attained wakelike shapes. The profiles close to the ports are characterized 
by a more narrow deficit and higher maximum velocities. The axial profile at $z / D=4.00$ is approximately constant. At $t / t_{\text {cyc }}=0.30$, the axial velocity deficit at $z / D=1.00$ and 1.50 has increased resulting in the formation of a central toroidal recirculation zone (CTRZ). It is generally accepted that the CTRZ is a form of vortex breakdown, i.e., an abrupt change in the structure of the vortex core, indicating a transition in flow state (Benjamin 1965; Escudier and Keller 1985; Lucca-Negro and O'Doherty 2001). For $t / t_{\mathrm{cyc}}=0.32-0.34$, the deficit becomes more narrow and the central recirculation zone disappears. The axial profile at $z / D=4.00$ has now also attained a wake-like shape. At $t / t_{\text {cyc }}=0.40$, the ports are closed and a significant change in the axial velocity profiles is observed. The profiles still have a wake-like shape, but the maximum velocities are significantly reduced, the deficit has become significantly wider, and negative centerline velocities are observed for all axial positions. For the remaining times during the standstill period $t / t_{\mathrm{cyc}}=0.50-1.00$, the centerline velocity is observed to change between positive and negative values. The nonzero axial velocities, observed during the piston standstill, are expected to be caused by large coherent swirl-induced structures that are moving up and down in the cylinder. The behavior is complex, resulting in, for example, positive centerline velocities at one axial position and negative centerline velocities at the next position (cf. Fig. $10, t / t_{\mathrm{cyc}}=0.60$ at $z / D=3.00$ and 4.00 ).

The unsteady behavior of the axial velocities is investigated further using the time-resolved LDA measurements. In Fig. 11, time series are shown for three measurement points, and in Online Resource 2-5, animations are presented showing the time-resolved radial profiles of the axial velocity. In addition to the reference case, animations are presented for cases with port angles of $\alpha=0^{\circ}, 10^{\circ}$, and $30^{\circ}$. In the $\alpha=30^{\circ}$ port angle case, the unsteady motion

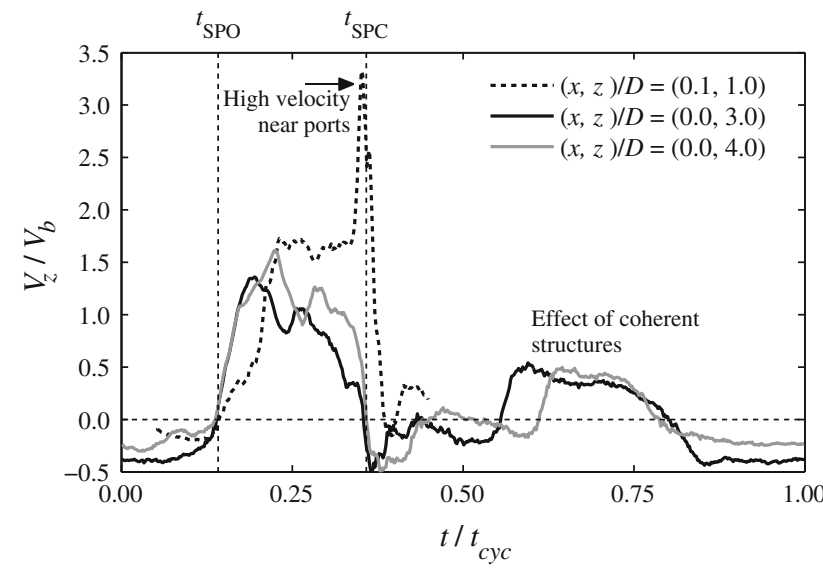

Fig. 11 LDA measurements of the phase-averaged axial velocity $V_{z} / V_{b}$ at three points in the cylinder during the piston standstill is more pronounced than in the $\alpha=20^{\circ}$ case, whereas the velocity is approximately zero during the entire standstill period for the nonswirling case $\left(\alpha=0^{\circ}\right)$. This shows that the unsteady structures observed during the standstill period is related to the in-cylinder swirl strength. The structures may result in cycle-to-cycle variations and affect combustion why further investigations must be carried out to obtain a more detailed understanding. Finally, the LDA measurements show that high axial velocities $V_{z} / V_{b} \approx 3.5$ occur locally in the bottom of the cylinder shortly before $t_{\mathrm{SPC}}$ (cf. Fig. 11).

\subsection{Streamlines}

To visualize the flow development, a series of streamline plots are presented in Fig. 12 together with the phase-

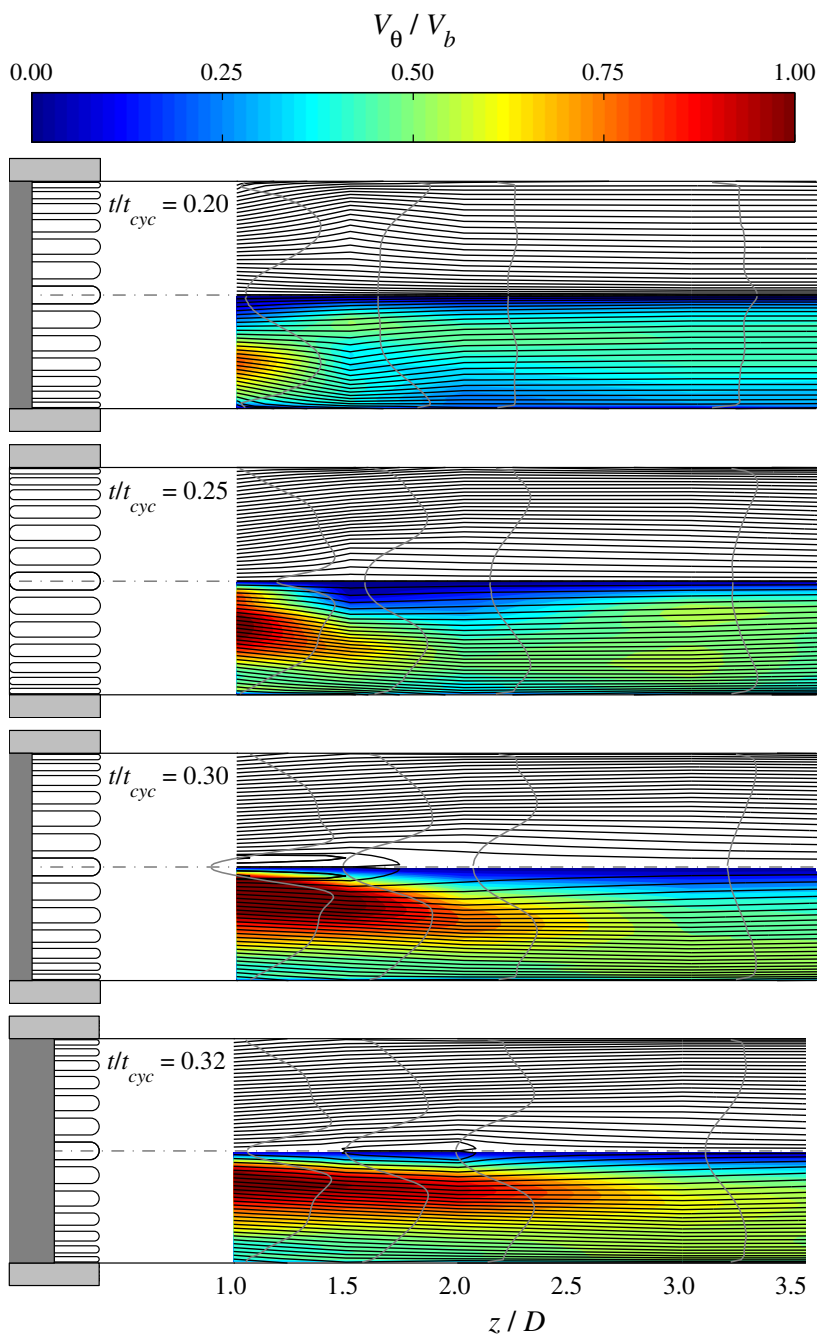

Fig. 12 Iso-contours of the stream function showing the development of the vortex breakdown. The colors in the lower half of the cylinder show the phase-averaged tangential velocity $V_{\theta} / V_{b}$ and the gray profiles show the axial velocity 
averaged tangential velocity. The stream function $\psi$ is calculated from the phase-averaged axial velocity by

$\psi(r, z)=\int_{0}^{r} \hat{r} V_{z}(\hat{r}, z) \mathrm{d} \hat{r}$

As the swirl intensity in the cylinder is increased by the incoming charge, the pressure at the centerline is reduced due to the centrifugal forces. The low pressure at the centerline retards the axial velocity, leading to the wakelike shape of the profiles. At $t / t_{\mathrm{cyc}}=0.30$, the pressure at the centerline is so low that the axial flow reverses. The resulting central recirculation zone is clearly visualized by the streamlines. At $t / t_{\mathrm{cyc}}=0.32$, the incoming swirl has reached $z / D \approx 3.0$, and the recirculation zone is reduced and has moved downstream, extending from approximately $z / D=1.5-2.0$.

\subsection{Phase-locked rms velocity profiles}

The radial profiles of phase-locked rms velocities $\left(V_{r, \mathrm{rms}}\right.$, $\left.V_{\theta, \text { rms }}, V_{z, \text { rms }}\right)$ are presented in Online Resource 1 for all measurement times and axial positions. The general trends in the profiles are found to be the same for the different velocity components. Examples of the rms profiles are presented in Fig. 13a, where the $V_{z, r m s}$-profiles are shown at four selected times during the cycle. The rms velocities at $t / t_{\mathrm{cyc}}=0.00$ are relatively low with an approximately uniform radial distribution. Near the axis, slightly higher values are observed which are expected to be the result of cycle-to-cycle variations of the instantaneous swirl center location.

At $t / t_{\text {cyc }}=0.25$, the ports are fully open and large rms velocities are observed in the bottom of the cylinder. The $z / D=1.00$ profile has a large and narrow peak at the cylinder axis. Inspection of the instantaneous velocity fields shows that there are three main contributions to the variations: (1) in-plane swirl center movement, (2) in-plane deformation of the vortex core (see e.g., Fig. 14d), and (3) intermittent appearance of the vortex breakdown, resulting in negative axial velocities in a small region at the swirl center.

At $t / t_{\mathrm{cyc}}=0.32$, the profiles at the first three axial positions also have a central peak, but a local minimum now exists at the very center. The local minimum is caused by a reduction of the swirl center movement, combined with the fact that the region with negative axial velocities has increased in size and appears at every instantaneous velocity field. Shortly after the scavenge port closing, at $t / t_{\text {cyc }}=0.40$, large rms velocities with an approximately uniform radial distribution are observed. This indicates that the high axial velocities observed just before port closing
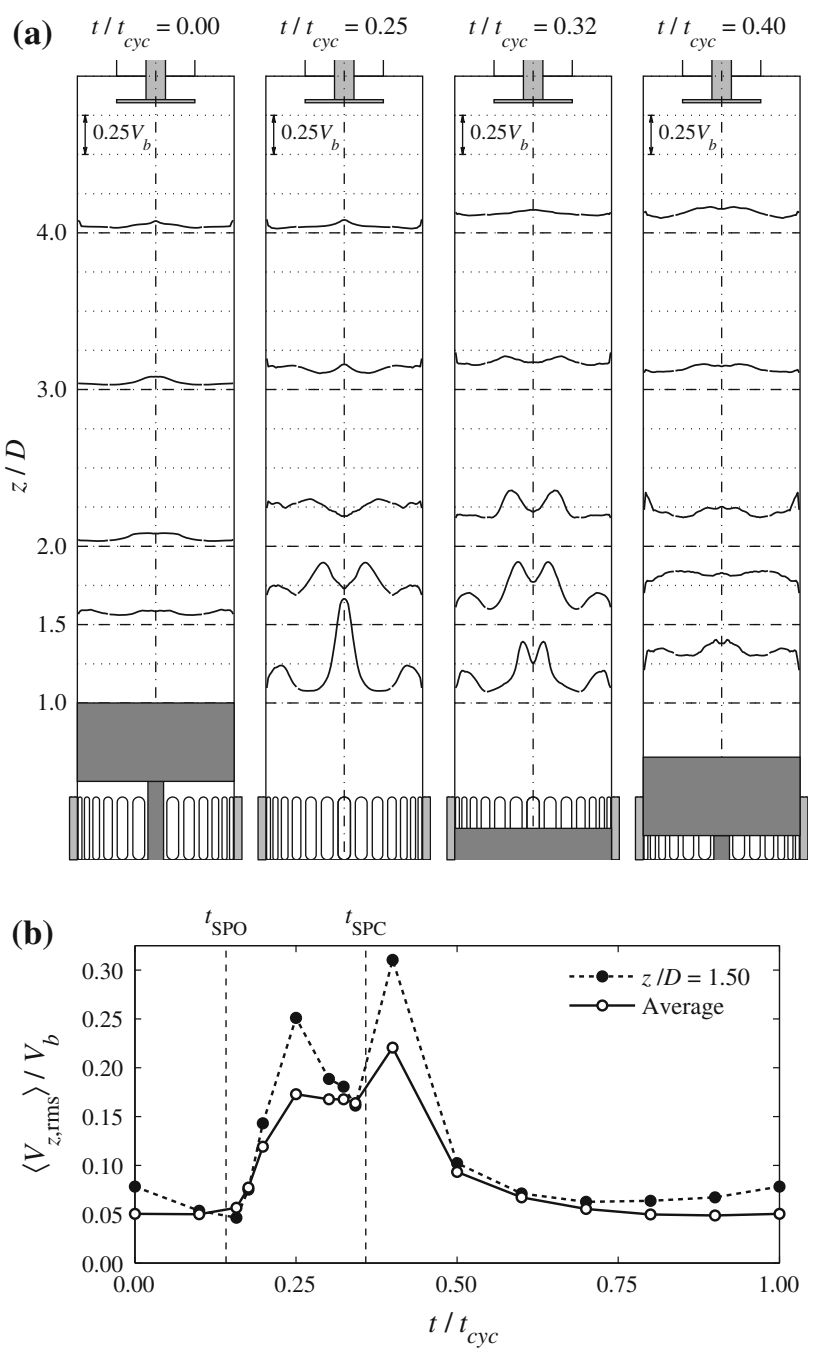

Fig. 13 a Phase-locked rms velocity profiles of the axial velocity component $V_{z, \text { rms }}$; b Mean cross-sectional rms velocity $\left\langle V_{z, \text { rms }}\right\rangle$

results in a strong excitation of the in-cylinder flow field, leading to large cycle-to-cycle variations.

The mean cross-sectional rms velocity $\left\langle V_{z, \text { rms }}\right\rangle$, obtained by integrating the radial profiles, is presented as a function of time in Fig. 13b. Two curves are presented in the figure: one curve corresponding to the $z / D=1.50$ plane, and one curve corresponding to the average of the five axial positions. The magnitude of the in-cylinder rms velocities at the cycle start is approximately $5 \%$ of $V_{b}$. After port opening, the rms velocity increases until the piston reaches bottom dead center at $t / t_{\mathrm{cyc}}=0.25$, indicating that the introduction of fresh air leads to large variations in the flow field. From bottom dead center to the scavenge port closing, the rms velocity decreases. It is expected that the reduction in rms velocities is the effect of the increased swirl strength which stabilizes the flow. At $t / t_{\mathrm{cyc}}=0.40$, shortly after the scavenge port closing, the cycle maximum rms velocity is observed showing that the port closing 

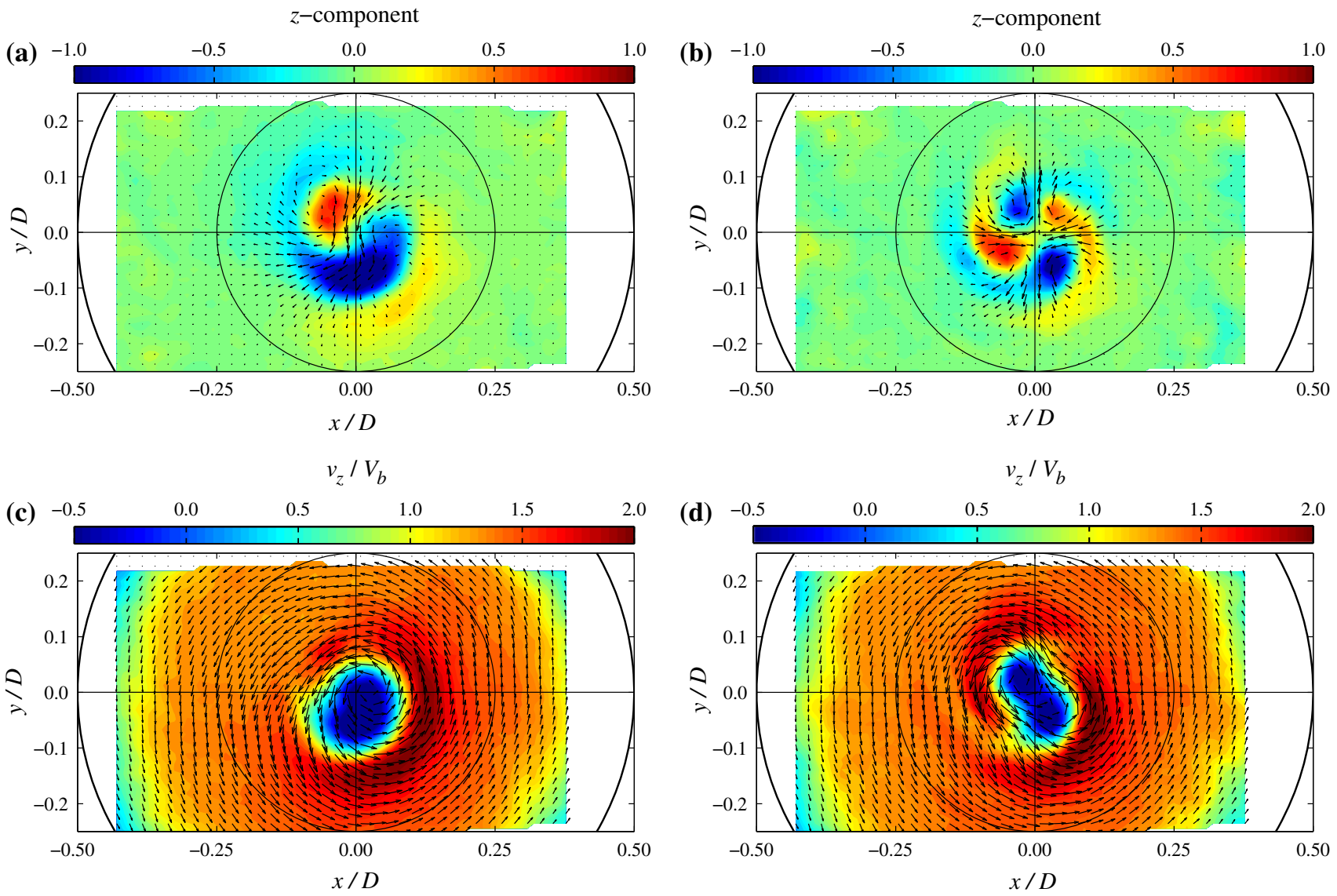

Fig. 14 Results from POD analysis at $z / D=1.00$ and $t / t_{\text {cyc }}=0.30$ : a mode $1 ; \mathbf{b}$ mode 3 ; $\mathbf{c}$ mode 1 added to the mean velocity field; $\mathbf{d}$ mode 3 added to the mean velocity field. The in-plane components are shown by vectors, and the $z$-component is shown by color

results in an strong excitation of the flow field. From $t / t_{\text {cyc }}=0.40-1.00$, the rms velocities show a decreasing trend. The curve for the $z / D=1.50$ position show a slight increase near the end of the cycle. The increase is expected to be caused by a large coherent structure that moves to the bottom of the cylinder. This is supported by the behavior of the tangential velocity profiles in Fig. 10 that show an increase in the gradient close to the center for the $z / D=$ 1.50 position at $t / t_{\mathrm{cyc}}=0.80$.

\subsection{Proper orthogonal decomposition analysis}

A snapshot proper orthogonal decomposition (POD) analysis (Sirovich 1987; Meyer et al. 2007) is performed to enhance the understanding of the flow dynamics and the spatial coherent structures. The analysis is performed for measurements acquired at $z / D=1.00$ for the times $t / t_{\text {cyc }}=\{0.25,0.30\}$, where larger measurement series, consisting of $n=250$ samples, are available.

The data series at $t / t_{\mathrm{cyc}}=0.30$ is particularly interesting as the vortex breakdown exists at this time during the cycle and the field of view cuts through the recirculation zone. In
Fig. 14, the first and the third POD mode are presented. To visualize the effects of the two modes, two additional plots are presented, where the modes are added to the mean field (cf. Fig. 3). At $t / t_{\mathrm{cyc}}=0.30$, the POD analysis shows that two pairs of dominating modes exist. The first mode pair (mode 1 and 2) constitutes $15 \%$ of the total energy, and the two modes are qualitatively the same except for a $90^{\circ}$ rotation around the cylinder axis. These modes are characterized by one region with a positive $z$-component and one region with a negative $z$-component placed symmetrically around the cylinder axis (Fig. 14a). In a polar coordinate system, this corresponds to an azimuthal mode with a period of one $(k=1)$. By comparing Fig. $14 \mathrm{c}$ with Fig. 3 , it is seen that mode 1 corresponds to a displacement of the vortex core. A superposition of mode 1 and 2 can therefore represent an arbitrary displacement of the vortex core. The first mode pair therefore corresponds to cycle-tocycle variations of the vortex core position.

The second mode pair (mode 3 and 4 ) constitutes $9 \%$ of the total energy, and the two modes are qualitatively the same except for a $45^{\circ}$ rotation around the cylinder axis (Fig. 14b). These modes are characterized by two regions 
Fig. 15 Probability density functions of velocity at $z / D=$ 1.00 and $t / t_{\mathrm{cyc}}=0.30$ : PDF of a radial velocity $v_{r} / V_{b}$ b tangential velocity $v_{\theta} / V_{b}$; c axial velocity $v_{z} / V_{b}$
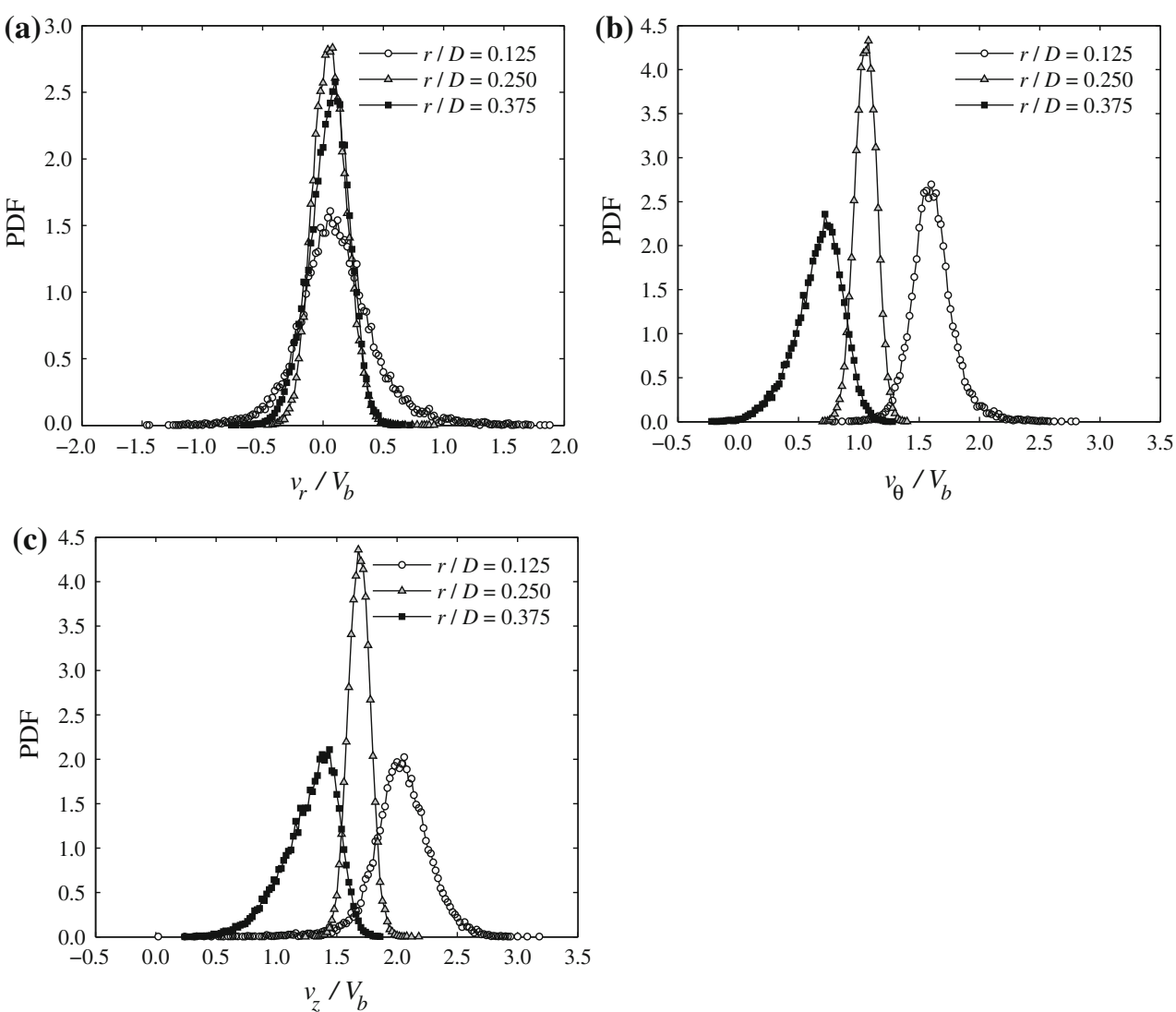

with positive $z$-component and two regions with negative $z$ component (Fig. 14b). This corresponds to an azimuthal mode with a period of two $(k=2)$. Depending on the energy of the mode at a given instance in time, the effect of the mode can be either an in-plane deformation of the vortex core or the formation of two separate swirl centers (Fig. 14d). A similar behavior was observed for the measurements through the vortex breakdown region in the steady-flow rate investigations (Ingvorsen et al. 2012, 2013). It has been shown, in both experimental and numerical studies of swirling flows, that double or triple helical structures often exist in the vortex breakdown region (see e.g., Ruith et al. 2003; Sørensen et al. 2009, 2011). It is therefore expected that the second mode pair (mode 3 and 4) represents a double helical structure existing in the vortex breakdown region.

Analysis of the data obtained at $t / t_{\text {cyc }}=0.25$ shows that a $k=1$ mode pair exist, however, in contrast to the results from $t / t_{\text {cyc }}=0.30$, no $k=2$ mode pair is found. This indicates that the double helical structure is formed later in the cycle, together with the vortex breakdown.

\subsection{Velocity PDFs}

To extend the use of the presented data for validation purposes in a large eddy simulation (LES) context, the probability density function (PDF) of the instantaneous velocities is presented. The PDF is computed for the velocity components expressed in the cylindrical coordinate system. The velocity components are therefore given by $v_{i}(r, z)$, where $i=1,2,3$ corresponds to $r, \theta, z$. However, since the experimental data are located in a Cartesian grid, it is necessary to define the velocity at a given radial coordinate $r_{1}$ as all the observations located in a thin annular region centered around $r_{1}$, i.e., $v_{i}\left(r_{1}\right)=v_{i}(r)$ for all $\left|r-r_{1}\right|<\Delta r$.

The PDFs are computed for the data obtained at $z / D=$ 1.00 and $t / t_{\text {cyc }}=0.30$, which is the same data that is used for the POD analysis ( $\Delta r / D=0.01$ is used). In Fig. 15, the PDFs of the three velocity components are shown for the radial positions $r / D=\{0.125,0.250,0.375\}$. In Table 2, the four main statical moments of the distributions are presented, i.e., the mean value $V$, the standard deviation $V_{\text {rms }}$, the skewness factor $S$, and the flatness factor (kurtosis) $F$.

It is noted that the mean velocity for the radial component $v_{r}$ is approximately constant and zero, whereas the mean velocity decreases as a function of the radial position for the $v_{\theta}$ and $v_{z}$ components. For all velocity components, the most narrow distribution (smallest $V_{\text {rms }}$ ) is observed at $r / D=0.250$. Furthermore, it is observed that the distributions at $r / D=0.250$ are nearly Gaussian, with 
Table 2 Statistical moments for the velocity distributions presented in Fig. 15
Mean $V$, standard deviation flatness factor $F$ $V_{\text {rms }}$, skewness factor $S$, and

\begin{tabular}{llllrl}
\hline$r / D$ & $i$ & $V_{i} / V_{b}$ & $V_{i, \mathrm{rms}} / V_{b}$ & \multicolumn{1}{c}{$S$} \\
\hline 0.125 & $r$ & 0.11 & 0.32 & 0.44 & 5.02 \\
0.125 & $\theta$ & 1.61 & 0.18 & 0.67 & 5.39 \\
0.125 & $z$ & 2.03 & 0.24 & -0.73 & 6.77 \\
0.250 & $r$ & 0.06 & 0.14 & 0.15 & 3.17 \\
0.250 & $\theta$ & 1.06 & 0.09 & -0.08 & 2.91 \\
0.250 & $z$ & 1.69 & 0.09 & 0.02 & 3.11 \\
0.375 & $r$ & 0.05 & 0.17 & -0.32 & 3.08 \\
0.375 & $\theta$ & 0.67 & 0.20 & -0.57 & 3.42 \\
0.375 & $z$ & 1.28 & 0.22 & -0.75 & 3.43 \\
\hline
\end{tabular}

a small skewness factor and a flatness factor of approximately 3.0. Closer to the wall, at $r / D=0.375$, all distributions show significant negative skewness, whereas the distributions closest to the center, $r / D=0.125$, are characterized by a high flatness factor.

\subsection{Comparison of dynamic and steady-flow case}

The tangential velocity profiles in the bottom of the cylinder at $t / t_{\mathrm{cyc}}=0.30$ have, as mentioned in Sect. 3.3, a shape similar to the profiles measured under steadyflow conditions. To investigate to which degree the flow under steady-flow conditions represents the flow under dynamic conditions, a comparison is carried out between the steady-flow profiles and the dynamic profiles. In Fig. 16, the comparison is shown for the axial positions $z / D=1.00$ and $z / D=2.00$ using the dynamic profiles from $t / t_{\text {cyc }}=0.30$. For $z / D=1.00$, it is observed that there is a good agreement between the steady-flow and dynamic profiles, with the best agreement seen between the tangential velocity profiles. The differences between the profiles are, however, found to increase as a function of the axial position, which can be seen from Fig. 16b. The tangential velocity for the steady-flow case shows a higher gradient near the center and has a maximum velocity approximately 1.5 times higher than the dynamic profile. It can thus be concluded that the flow in a uniflow-scavenge engine cannot be assumed to be quasisteady, except in the bottom of the cylinder at times when the ports are almost fully open. This conclusion does, to some degree, contradict the findings of earlier investigators, claiming that the scavenging characteristics in uniflow-scavenged engines can be predicted from steady-flow investigations (see e.g., Ohigashi et al. 1960; Percival 1955). It is also worth noting that the wellestablished Jante method, used for optimizing the scavenge design, is based on steady-flow investigations (Heywood and Sher 1999).

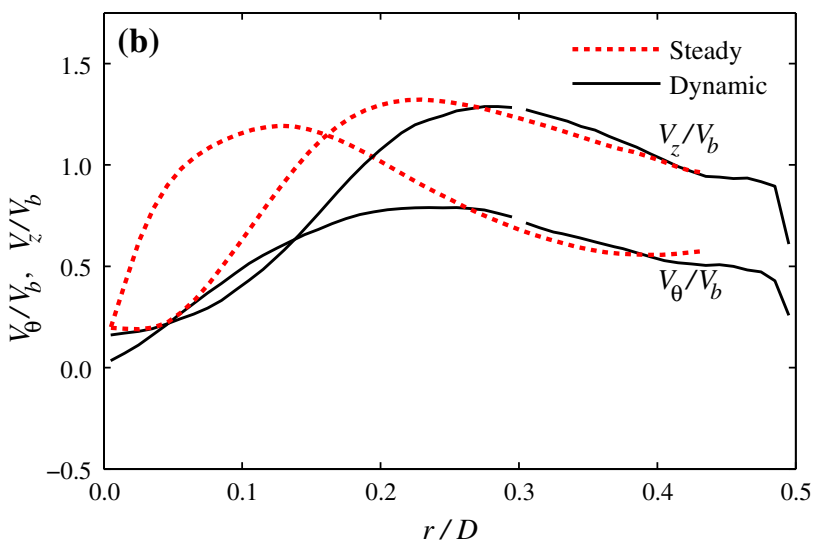

Fig. 16 Comparison of radial velocity profiles for the steady-flow case (Ingvorsen et al. 2013) and the dynamic case at $t / t_{\mathrm{cyc}}=0.30$ for

\subsection{Angular momentum}

The generation and decay of swirl are investigated by calculating the angular momentum from the radial profiles of tangential velocity at the different axial positions. The angular momentum per unit length is given by

$\Gamma(z, t)=\int_{A_{c y l}} \rho V_{\theta} r \mathrm{~d} A=2 \pi \int_{0}^{D / 2} \rho V_{\theta}(r) r^{2} \mathrm{~d} r$

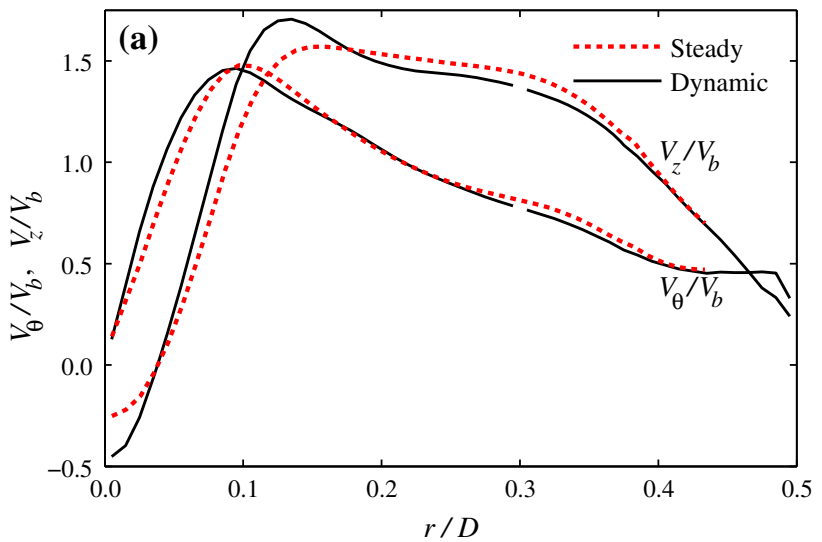
axial positions a $z / D=1.00 ; \mathbf{b} z / D=2.00$ 


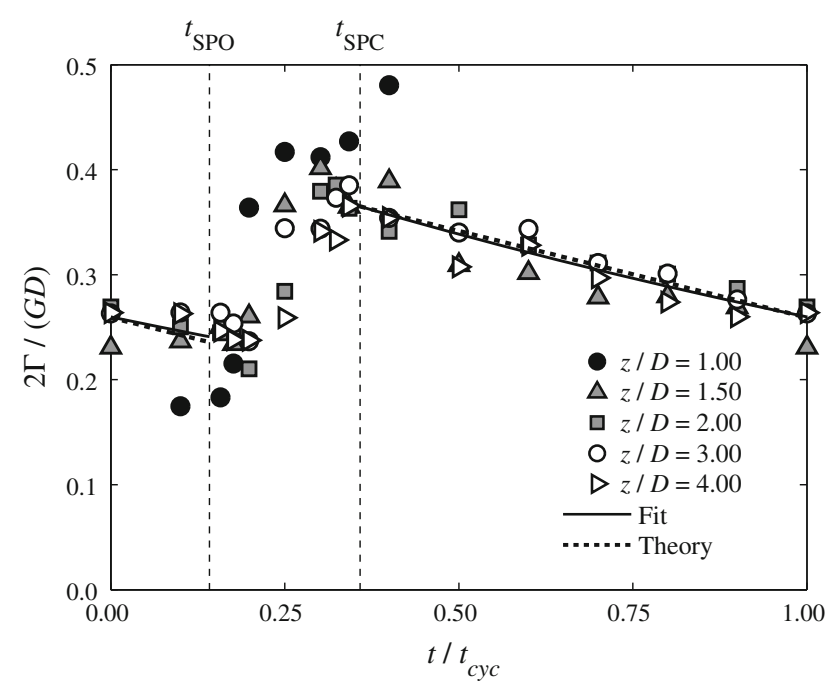

Fig. 17 Normalized angular momentum as a function of time for different axial positions. The solid line shows fit with exponential decay

The angular momentum for the five axial positions is presented as a function of time in Fig. 17. In the figure, the angular momentum is normalized using the cylinder radius and the axial momentum at $t / t_{\text {cyc }}=0.25$ which is given by $G=\rho A_{c y l} V_{b}$. After scavenge port opening, a general increase in angular momentum is observed. The axial positions closest to the scavenge ports show the increase first and also show the highest levels of angular momentum. From scavenge port closing until scavenge port opening, the angular momentum shows a monotonic decreasing trend and a reduction in the axial variation. Confined turbulent swirling flows are known to show an exponential decay (Kitoh 1991; Kreith and Sonju 1965). The decay rate of the swirl $\lambda$ is estimated by fitting an exponential function of the form $\Gamma(t)=\Gamma_{0} \exp \left(-\lambda t / t_{\text {cyc }}\right)$ to the angular momentum from port closing to port opening. The decay rate is found to be $\lambda=0.53$ corresponding to a reduction of $34 \%$ from scavenge port closing to scavenge port opening.

The observed reduction in angular momentum can be compared to simple theoretical predictions based on friction formulas developed for the flow over a flat plate (Heywood 1988). The decay rate of angular momentum is equal to the torque from wall friction. The torque from friction at the cylinder wall (friction at the piston surface and cylinder head is neglected) is given by $T_{f}=\frac{1}{2} D O_{c y l} \tau$, where $O_{\text {cyl }}=\pi D$ is the circumference of the cylinder and $\tau$ is the wall shear stress. The wall shear stress can be evaluated using the friction factor $C_{f}$ and Reynolds number $R e_{\omega}$ as given below (Heywood 1988)

$$
\begin{aligned}
& \tau=\frac{1}{2} \rho\left(\omega_{s} D / 2\right)^{2} C_{f} \\
& C_{f}=0.037 \gamma \operatorname{Re}_{\omega}^{-0.2} \\
& \operatorname{Re}_{\omega}=\rho\left(D \omega_{s} / 2\right)(\pi D) / \mu
\end{aligned}
$$

Here, $\gamma$ is an empirical constant accounting for the difference between the flat plate and the curved cylinder wall $(\lambda=1.5)$ cf. Davis and Kent (1979), and $\omega_{s}=\Gamma /\left(\frac{\pi}{32} \rho D^{4}\right)$ is the angular velocity of the equivalent solid body rotation. Assuming a constant torque based on the angular momentum at $t_{\mathrm{SPC}}$, a reduction of $35 \%$ is predicted, which is in excellent agreement with the present measurements.

\section{Conclusions}

The turbulent swirling flow in a dynamic scale model of a low-speed two-stroke uniflow-scavenged marine diesel engine is investigated experimentally. The flow is characterized using phase-locked stereoscopic particle image velocimetry (PIV) and laser Doppler anemometry (LDA). Radial velocity profiles are generated from the PIV data, and the accuracy of the profiles is evaluated through repeated measurements and comparison with LDA. The main conclusions of the work are as follows:

- A database of experimental results suitable for CFD validation purposes is established. The experimental velocity profiles are shown to have an uncertainty corresponding to approximately $3 \%$ of the bulk velocity at fully open ports.

- The temporal development of the in-cylinder flow is shown to be complex. In the period when the ports are closed, the tangential velocity profiles are characterized by a central region with approximately solid body rotation and an annular region with approximately constant velocity. In the same period, the axial velocity profiles show nonzero velocities, indicating that large unsteady swirl-induced flow structures exist.

- Comparison of profiles obtained under steady-flow and dynamic conditions shows that the scavenge flow cannot be assumed to be quasi-steady.

- The swirl strength shows an exponential decay from scavenge port closing to scavenge port opening, corresponding to a reduction of $34 \%$, which is found to be in good agreement with theoretical predictions.

Acknowledgments Financial funding from the Danish Agency for Science Technology and Innovation (Grants No. 09-070608, MAN Diesel \& Turbo) is greatly acknowledged. 


\section{References}

Benjamin TB (1965) Significance of the vortex breakdown phenomenon. J Basic Eng 87:518-522

Blair GP (1996) Design and simulation of two-stroke engines. SAE International, 400 Commonwealth Drive, Warrendale

Davis GC, Kent JC (1979) Comparison of model calculations and experimental measurements of the bulk cylinder flow processes in a motored PROCO engine. SAE Tech Paper Ser Paper No. 790290

Diwakar R (1987) Three-dimensional modeling of the in-cylinder gas exchange processes in a uniflow-scavenged two-stroke engine. SAE Tech Paper Ser Paper No. 870596

Escudier MP, Keller JJ (1985) Recirculation in swirling flow: a manifestation of vortex breakdown. AIAA J 23(1):111-116

Goldsborough SS, Blarigan PV (2003) Optimizing the scavenging system for a two-stroke cycle, free piston engine for high efficiency and low emissions: a computational approach. SAE Tech Paper Ser 1:1-22

Haider S, Schnipper T, Obeidat A, Meyer KE, Okulov VL, Mayer S, Walther JH (2013) PIV study of the effect of piston position on the in-cylinder swirling flow during the scavenging process in large two-stroke marine diesel engines. J Mar Sci Technol 18:133-143. doi:10.1007/s00773-012-0192-z

Heywood JB (1988) Internal combustion engine fundamentals. McGraw Hill Inc, New York

Heywood JB, Sher E (1999) The two-stroke cycle engine: its development, operation, and design. Taylor \& Francis, London

Hult J, Mayer S (2013) A methodology for laser diagnostics in largebore marine two-stroke diesel engines. Meas Sci Technol 24(4):1-10

Ingvorsen KM, Meyer KE, Schnipper T, Walther JH, Mayer S (2012) Swirling flow in model of large two-stroke diesel engine. In: 16th International symposium on applications of laser techniques to fluid mechanics, Lisbon, Portugal

Ingvorsen KM, Meyer KE, Walther JH, Mayer S (2013) Turbulent swirling flow in a model of a uniflow-scavenged two-stroke engine. Exp Fluids 54(3): 1494

Jakirlić S, Hanjalić K, Tropea C (2002) Modeling rotating and swirling turbulent flows: a perpetual challenge. AIAA J 40(10):1984-1996

Joenson TV (2011) Numerical simulation of the flow in a model diesel engine. Master's thesis, Technical University of Denmark

Kitoh O (1991) Experimental study of turbulent swirling flow in a straight pipe. J Fluid Mech 225:445-479

Kreith F, Sonju OK (1965) The decay of a turbulent swirl in a pipe. J Fluid Mech 22(2):257-271

Lucca-Negro O, O'Doherty T (2001) Vortex breakdown: a review. Prog Energy Combust Sci 27:431-481
Meyer KE, Pedersen JM, Özcan O (2007) A turbulent jet in crossflow analyzed with proper orthogonal decomposition. J Fluid Mech 583:199-227

Nakagawa H, Kato S, Tateishi M, Adachi T, Tsujimura H, Nakashima M (1990) Airflow in the cylinder of a 2-stoke cycle uniflow scavenging diesel engine during compression stroke. Jpn Soc Mech Eng 33(3):591-598

Obeidat A, Schnipper T, Ingvorsen KM, Haider S, Meyer KE, Mayer S, Walther JH (2014) Large eddy simulations of the influence of piston position on the swirling flow in a model two-stroke diesel engine. Int J Num Method Heat Fluid Flow 24(2):325-341

Ohigashi S, Kashiwada Y, Achiwa J (1960) Scavenging the 2-stroke engine-effect of inlet port-angle on scavenging process of a through scavenging system. Jpn Soc Mech Eng 3(9):130-136

Percival WH (1955) Method of scavenging analysis for 2-stroke-cycle diesel cylinders. SAE Trans 63:737-751

Ruith MR, Chen P, Meiburg E, Maxworthy T (2003) Threedimensional vortex breakdown in swirling jets and wakes: direct numerical simulation. J Fluid Mech 486:331-378

Schweitzer PH (1949) Scavenging of two-stroke cycle diesel engines. Macmillan Publishing Company, New York

Sher E, Hossain I, Zhang Q, Winterbone DE (1991) Calculation and measurements in the cylinder of a two-stroke uniflow-scavenged engine under steady flow conditions. Exp Therm Fluid Sci 4:418-431

Sigurdsson E, Ingvorsen KM, Jensen MV, Mayer S, Matlok S, Walther JH (2014) Numerical analysis of scavenge flow and convective heat transfer in large two-stroke marine diesel engines. Appl Energy 123:37-46

Sirovich L (1987) Turbulence and the dynamics of coherent structures. Part I: coherent structures. Quart Appl Math 45(3):561-570

Sørensen JN, Gelfgat AY, Naumov IV, Mikkelsen RM (2009) Experimental and numerical results on three-dimensional instabilities in a rotating disk-tall cylinder flow. Phys Fluids 21(5):054,102

Sørensen JN, Naumov IV, Okulov VL (2011) Multiple helical modes of vortex breakdown. J Fluid Mech 683:430-441

Steenbergen W, Voskamp J (1998) The rate of decay of swirl in turbulent pipe flow. Flow Meas Instrum 9(2):67-78

Sung NW, Patterson DJ (1982) Air motion in a two stroke engine cylinder-the effects of exhaust geometry. SAE Trans, pp 2534-2544, Paper No. 820751

Uzkan T (1988) The effects of engine speed on the scavenging characteristics of a two-cycle engine. J Eng Gas Turbines Power 110:523-530

Young HD, Freedman RA (2004) Sears and Zemansky's university physics: with modern physics, 11th edn. Addison-Wesley Publishing Company, San Fransico 